TRANSACTIONS OF THE

AMERICAN MATHEMATICAL SOCIETY

Volume 365, Number 7, July 2013, Pages 3613-3641

S 0002-9947(2013)05717-0

Article electronically published on February 28, 2013

\title{
KUMJIAN-PASK ALGEBRAS OF HIGHER-RANK GRAPHS
}

\author{
GONZALO ARANDA PINO, JOHN CLARK, ASTRID AN HUEF, AND IAIN RAEBURN
}

Abstract. We introduce higher-rank analogues of the Leavitt path algebras, which we call the Kumjian-Pask algebras. We prove graded and Cuntz-Krieger uniqueness theorems for these algebras, and analyze their ideal structure.

\section{INTRODUCTION}

The $C^{*}$-algebras of infinite directed graphs were first studied in the 1990s 23 , 22 as generalizations of the Cuntz-Krieger algebras of finite $\{0,1\}$-matrices [12]. The Leavitt path algebras, which are a purely algebraic analogue of graph $C^{*}$ algebras, were first studied around 2005 [1, 5]. Both families of algebras have been intensively studied by a broad range of researchers, both now have substantial structure theories, and both have proved to be rich sources of interesting examples.

Higher-rank analogues of the Cuntz-Krieger algebras arose first in work of Robertson and Steger [33, 34, and shortly afterwards Kumjian and Pask [21] introduced higher-rank graphs (or $k$-graphs) to provide a visualisable model for Robertson and Steger's algebras. The higher-rank graph $C^{*}$-algebras constructed in [21] have generated a great deal of interest among operator algebraists (for example, [13, 20, 27, 36, 39]), and have broadened the class of $C^{*}$-algebras that can be realized as graph algebras [14, 21, 25, 26. Here we introduce and study an analogue of Leavitt path algebras for higher-rank graphs. We propose to call these new algebras the Kumjian-Pask algebras.

For operator algebraists, there is a well-trodden path for studying new analogues of Cuntz-Krieger algebras, which was developed in [19] and [9], and which was followed in the first four chapters of [28, for example. First, one constructs an algebra which is universal among $C^{*}$-algebras generated by families of partial isometries satisfying suitable Cuntz-Krieger relations. Next, one proves uniqueness theorems which say when a representation of this algebra is injective: there should be a gauge-invariant uniqueness theorem, which works without extra hypotheses on the graph, and a Cuntz-Krieger uniqueness theorem, which has a stronger conclusion but requires an aperiodicity hypothesis. Then one hopes to use these theorems to analyze the ideal structure.

Tomforde tramped this path for the Leavitt path algebras over fields 37, and more recently has retramped it for Leavitt path algebras over commutative rings [38. We will use the same path to study the Kumjian-Pask algebras of row-finite

Received by the editors June 21, 2011 and, in revised form, October 4, 2011.

2010 Mathematics Subject Classification. Primary 16W50; Secondary 46L05.

Key words and phrases. Leavitt path algebra, Cuntz-Krieger algebra, higher-rank graph, graph algebra.

The results in this paper were obtained during a working seminar at the University of Otago. The authors thank the other participants for their comments and input, and especially Jon Brown, Iain Dangerfield and Robbie Hazlewood. 
$k$-graphs without sources. There are satisfactory $C^{*}$-algebraic uniqueness theorems for larger families of $k$-graphs, but they can be very complicated to work with (look at the proof of Cuntz-Krieger uniqueness in [31, for example). So for a first pass it seems sensible to stick to the row-finite case, which covers most of the interesting examples. We follow 38, in allowing coefficients in an arbitrary commutative ring $R$ with identity 1 .

We begin with a section on background material. We recall from [21] some elementary facts about higher-rank graphs and their infinite path spaces, and also discuss some basic properties of gradings on free algebras which we couldn't find in suitable form in the literature. Then in 93 , we describe our Kumjian-Pask relations for a row-finite $k$-graph $\Lambda$ without sources, and construct the Kumjian-Pask algebra $\mathrm{KP}_{R}(\Lambda)$ as a quotient of the free $R$-algebra on the set of paths in $\Lambda$. Because the Kumjian-Pask relations are substantially more complicated for $k$-graphs, we have had to be quite careful with this construction, and in particular with the existence of the $\mathbb{Z}^{k}$-grading on $\mathrm{KP}_{R}(\Lambda)$.

In 84 , we prove a graded-uniqueness theorem and a Cuntz-Krieger uniqueness theorem for $\mathrm{KP}_{R}(\Lambda)$. We have used similar arguments to those of [38, $\left.\S \S 5-6\right]$, but, partly because we are only interested in the row-finite case, we have been able to streamline the arguments and find a common approach to the two theorems. In particular, we were able to bypass the complicated induction arguments used in [38. As the main hypothesis in our Cuntz-Krieger uniqueness theorem we use the "finite-path formulation" of aperiodicity due to Robertson and Sims 32 .

In $\S \$ 5$ and 6, we investigate the ideal structure of $\mathrm{KP}_{R}(\Lambda)$. The first step is to describe the graded ideals, which we do in Theorem [5.1] as in [38, to get the usual description of ideals in terms of saturated hereditary subsets of vertices (which goes back to Cuntz [11]), we have to restrict attention to a class of "basic ideals". We then give an analogue of Conditions (II) of [11] and (K) of [23] which ensures that every basic ideal is graded, and describe the $k$-graphs for which $\operatorname{KP}_{R}(\Lambda)$ is "basically simple". Then in $\sqrt[6]{6}$, we find necessary and sufficient conditions for $\mathrm{KP}_{R}(\Lambda)$ to be simple in the more conventional sense. This last result is new even for 1-graphs. We discuss examples and applications in $₫ 7$.

\section{BACKGROUND}

We write $\mathbb{N}$ for the set of natural numbers, including 0 . Let $k$ be a positive integer. For $m, n \in \mathbb{N}^{k}, m \leq n$ means $m_{i} \leq n_{i}$ for $1 \leq i \leq k$ and $m \vee n$ denotes the pointwise maximum. We denote the usual basis in $\mathbb{N}^{k}$ by $\left\{e_{i}\right\}$.

In a category $\mathcal{C}$ with objects $\mathcal{C}^{0}$, we identify objects $v \in \mathcal{C}^{0}$ with their identity morphisms $\iota_{v}$, and write $\mathcal{C}$ for the set of morphisms; we write $s$ and $r$ for the domain and codomain maps from $\mathcal{C}$ to $\mathcal{C}^{0}$, and usually denote the composition of morphisms by juxtaposition.

A directed graph $E=\left(E^{0}, E^{1}, r, s\right)$ consists of countable sets $E^{0}$ and $E^{1}$ and functions $r, s: E^{1} \rightarrow E^{0}$. As usual, we think of the elements of $E^{0}$ as vertices and the elements $e$ of $E^{1}$ as edges from $s(e)$ to $r(e)$. Because we are going to be talking about higher-rank graphs, where a juxtaposition $\mu \nu$ stands for the composition of morphisms $\mu$ and $\nu$ with $s(\mu)=r(\nu)$, we use the conventions of [28] for paths in $E$. Thus a path of length $|\mu|:=n$ in $E$ is a string $\mu=\mu_{1} \cdots \mu_{n}$ of edges $\mu_{i}$ with $s\left(\mu_{i}\right)=r\left(\mu_{i+1}\right)$ for all $i$, and the path has source $s(\mu):=s\left(\mu_{n}\right)$ and range $r(\mu):=r\left(\mu_{1}\right)$. 
2.1. Higher-rank graphs. For a positive integer $k$, we view the additive semigroup $\mathbb{N}^{k}$ as a category with one object. Following Kumjian and Pask [21, a graph of rank $k$ or $k$-graph is a countable category $\Lambda=\left(\Lambda^{0}, \Lambda, r, s\right)$ together with a functor $d: \Lambda \rightarrow \mathbb{N}^{k}$, called the degree map, satisfying the following factorization property: if $\lambda \in \Lambda$ and $d(\lambda)=m+n$ for some $m, n \in \mathbb{N}^{k}$, then there are unique $\mu, \nu \in \Lambda$ such that $d(\mu)=m, d(\nu)=n$, and $\lambda=\mu \nu$.

The motivating example is:

Example 2.1. Consider a directed graph $E=\left(E^{0}, E^{1}, r, s\right)$. Then the path category $P(E)$ has object set $E^{0}$, and the morphisms in $P(E)$ from $v \in E^{0}$ to $w \in E^{0}$ are finite paths $\mu$ with $s(\mu)=v$ and $r(\mu)=w$; composition is defined by concatenation, and the identity morphisms obtained by viewing the vertices as paths of length 0 . With the degree functor $d: \mu \mapsto|\mu|$, the path category $(P(E), d)$ is a 1-graph.

With this example in mind, we make some conventions. If $\lambda \in \Lambda$ satisfies $d(\lambda)=$ 0 , the identities $\iota_{r(\lambda)} \lambda=\lambda=\lambda \iota_{s(\lambda)}$ and the factorization property imply that $\iota_{r(\lambda)}=\lambda=\iota_{s(\lambda)}$; thus $v \mapsto \iota_{v}$ is a bijection of $\Lambda^{0}$ onto $d^{-1}(0)$. Then for $n \in \mathbb{N}^{k}$, we write $\Lambda^{n}:=d^{-1}(n)$, and call the elements $\lambda$ of $\Lambda^{n}$ paths of degree $n$ from $s(\lambda)$ to $r(\lambda)$. For $v \in \Lambda^{0}$ we write $v \Lambda^{n}$ or $v \Lambda$ for the sets of paths with range $v$ and $\Lambda^{n} v$ or $\Lambda v$ for paths with source $v$.

To visualise a $k$-graph $\Lambda$, we think of the object set $\Lambda^{0}$ as the vertices in a directed graph, choose $k$ colours $c_{1}, \ldots, c_{k}$, and then for each $\lambda \in \Lambda^{e_{i}}$, we draw an oriented edge of colour $c_{i}$ from $s(\lambda)$ to $r(\lambda)$. This coloured directed graph $E$ is called the skeleton of $\Lambda$. When $k=1$, the skeleton is an ordinary directed graph, and completely determines the 1-graph: indeed, the factorization property allows us to write each morphism $\lambda$ of degree $n$ uniquely as the composition $\lambda_{1} \circ \lambda_{2} \circ \cdots \circ \lambda_{n}$ of $n$ morphisms of degree 1 , and then the map which takes $\lambda$ to the path $\lambda_{1} \lambda_{2} \cdots \lambda_{n}$ is an isomorphism of $\Lambda$ onto $P(E)$. When $k>1$, the skeleton does not always determine the $k$-graph. To discuss this issue, we need some examples.

Example 2.2. Let $\Omega_{k}^{0}:=\mathbb{N}^{k}, \Omega_{k}:=\left\{(p, q) \in \mathbb{N}^{k} \times \mathbb{N}^{k}: p \leq q\right\}$, define $r, s: \Omega_{k} \rightarrow$ $\Omega_{k}^{0}$ by $r(p, q):=p$ and $s(p, q):=q$, define composition by $(p, q)(q, r)=(p, r)$, and define $d: \Omega_{k} \rightarrow \mathbb{N}^{k}$ by $d(p, q):=q-p$. Then $\Omega_{k}=\left(\Omega_{k}, r, s, d\right)$ is a $k$-graph.

Similarly, for $m \in \mathbb{N}^{k}$ we define $\Omega_{k, m}^{0}:=\left\{p \in \mathbb{N}^{k}: p \leq m\right\}$ and $\Omega_{k, m}=\{(p, q) \in$ $\left.\Omega_{k, m}^{0} \times \Omega_{k, m}^{0}: p \leq q\right\}$, and then with the same $r, s$ and $d, \Omega_{k, m}$ is a $k$-graph. The skeleton of $\Omega_{2,(3,2)}$, for example, is

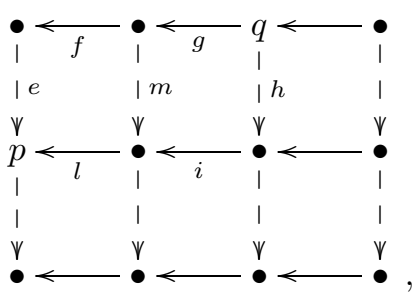

where the solid arrows are blue, say, and the dashed ones are red. We think of the paths as rectangles: for example, the path $(p, q)$ with source $q$ and range $p$ would be the $2 \times 1$ rectangle in the top left, and the different routes ef $g, l m g$, lih from $q$ to $p$ represent the different factorizations of $(p, q)$. 
The factorization property in a $k$-graph $\Lambda$ sets up bijections between the $c_{i} c_{j}$ coloured paths of length 2 and the $c_{j} c_{i}$-coloured paths, and we think of each pair as a commutative square in the skeleton. A theorem of Fowler and Sims [16] says that this collection $\mathcal{C}$ of commutative squares determines the $k$-graph; a path of degree $(3,2)$, for example, is obtained by pasting a copy of (2.1) around the skeleton in such a way that the colours are preserved and each constituent square is commutative. When $k=2$, every collection $\mathcal{C}$ which includes each $c_{i} c_{j}$-coloured path exactly once determines a 2-graph with the given skeleton [21, §6]; for $k \geq 3$, the collection $\mathcal{C}$ has to satisfy an extra associativity condition. (For a discussion with some pictures, see [30.)

A $k$-graph $\Lambda$ is row-finite if $v \Lambda^{n}$ is finite for every $v \in \Lambda^{0}$ and $n \in \mathbb{N}^{k} ; \Lambda$ has no sources if $v \Lambda^{n}$ is nonempty for every $v \in \Lambda^{0}$ and $n \in \mathbb{N}^{k}$. In this paper we are interested only in row-finite $k$-graphs without sources.

2.2. The infinite path space. Suppose that $\Lambda$ is a row-finite $k$-graph without sources. Following [21, §2], an infinite path in $\Lambda$ is a degree-preserving functor $x: \Omega_{k} \rightarrow \Lambda$. We denote the set of all infinite paths by $\Lambda^{\infty}$. Since we identify the object $m \in \Omega_{k}$ with the identity morphism $(m, m)$ at $m$, we write $x(m)$ for the vertex $x(m, m)$. Then the range of an infinite path $x$ is the vertex $r(x):=x(0)$, and we write $v \Lambda^{\infty}:=r^{-1}(v)$.

Remark 2.3. To motivate this definition, notice that an ordinary path $\lambda \in \Lambda^{n}$ gives a functor $f_{\lambda}: \Omega_{k, n} \rightarrow \Lambda$. To see this, take $0 \leq p \leq q \leq n$, use the factorization property to see that there are unique paths $\lambda^{\prime} \in \Lambda^{p}, \lambda^{\prime \prime} \in \Lambda^{q-p}$ and $\lambda^{\prime \prime \prime} \in \Lambda^{n-q}$ such that $\lambda=\lambda^{\prime} \lambda^{\prime \prime} \lambda^{\prime \prime \prime}$, and then define $f_{\lambda}(p, q):=\lambda(p, q):=\lambda^{\prime \prime}$. The map $\lambda \mapsto f_{\lambda}$ is a bijection from $\Lambda^{n}$ onto the set of degree-preserving functors from $\Omega_{k, n}$ to $\Lambda$, 30 , Examples 2.2(ii)].

Since $\Lambda$ has no sources, every vertex receives paths of arbitrarily large degrees, and the following lemma from 21] tells us that every vertex receives infinite paths.

Lemma 2.4 ([21, Remarks 2.2]). Suppose that $n(i) \leq n(i+1)$ in $\mathbb{N}^{k}$, that $n(i)_{j} \rightarrow$ $\infty$ in $\mathbb{N}$ for $1 \leq j \leq k$, and that $\lambda_{i} \in \Lambda^{n(i)}$ satisfy $\lambda_{i+1}(0, n(i))=\lambda_{i}$. Then there is a unique $y \in \Lambda^{\infty}$ such that $y(0, n(i))=\lambda_{i}$.

The next lemma, also from [21, §2], tells us that we can compose infinite paths with finite ones, and that there is a converse factorization process. The path $x(n, \infty)$ in part (b) was denoted $\sigma^{n}(x)$ in [21].

Lemma 2.5. (a) Suppose that $\lambda \in \Lambda$ and $x \in \Lambda^{\infty}$ satisfy $s(\lambda)=r(x)$. Then there is a unique $y \in \Lambda^{\infty}$ such that $y(0, n)=\lambda x(0, n-d(\lambda))$ for $n \geq d(\lambda)$; we then write $\lambda x:=y$.

(b) For $x \in \Lambda^{\infty}$ and $n \in \mathbb{N}^{k}$, there exist unique $x(0, n) \in \Lambda^{n}$ and $x(n, \infty) \in \Lambda^{\infty}$ such that $x=x(0, n) x(n, \infty)$.

2.3. Graded rings. Let $G$ be an additive abelian group. A ring $A$ is $G$-graded if there are additive subgroups $\left\{A_{g}: g \in G\right\}$ of $A$ such that $A_{g} A_{h} \subset A_{g+h}$ and every nonzero $a \in A$ can be written in exactly one way as a finite sum $\sum_{g \in F} a_{g}$ of nonzero elements $a_{g} \in A_{g}$. The elements of $A_{g}$ are homogeneous of degree $g$, and $a=\sum_{g \in F} a_{g}$ is the homogeneous decomposition of $a$. If $A$ and $B$ are $G$-graded rings, a homomorphism $\phi: A \rightarrow B$ is graded if $\phi\left(A_{g}\right) \subset B_{g}$ for all $g \in G$. 
Suppose that $A$ is $G$-graded by $\left\{A_{g}: g \in G\right\}$. An ideal $I$ in $A$ is a graded ideal if $\left\{I \cap A_{g}: g \in G\right\}$ is a grading of $I$. If $I$ is graded and $q: A \rightarrow A / I$ is the quotient map, then $A / I$ is $G$-graded by $\left\{q\left(A_{g}\right): g \in G\right\}$. To check that an ideal $I$ is graded, it suffices (by the uniqueness of homogeneous decompositions in $A$ ) to check that every element of $I$ is a sum of elements in $\bigcup_{g \in G}\left(I \cap A_{g}\right)$. Every ideal $I$ which is generated by a set $S$ of homogeneous elements is graded: to see this, it suffices by linearity and the previous observation to check that every element of

$$
\left\{a_{g} x b_{h}: a_{g} \in A_{g}, x \in S, b_{h} \in A_{h}\right\}
$$

belongs to some $I \cap A_{k}$, and this is easy.

Let $R$ be a commutative ring with identity 1 . For a nonempty set $Y$, we view the free $R$-module $\mathbb{F}_{R}(Y)$ with basis $Y$ as the set of formal sums $\sum_{y \in Y} r_{y} y$ in which all but finitely many coefficients $r_{y}$ are zero; we view the elements $y \in Y$ as elements of $\mathbb{F}_{R}(Y)$ by writing them as sums $\sum_{x} r_{x} x$ where $r_{x}=0$ for $x \neq y$ and $r_{y}=1$. For a nonempty set $X$, we let $w(X)$ be the set of words $w$ from the alphabet $X$, and we write $|w|$ for the length of $w$, so that $w=w_{1} w_{2} \cdots w_{|w|}$ for some $w_{i} \in X$. Then the free $R$-module $\mathbb{F}_{R}(w(X))$ is an $R$-algebra with multiplication given by

$$
\left(\sum_{w \in w(X)} r_{w} w\right)\left(\sum_{y \in w(X)} s_{y} y\right)=\sum_{z \in w(X)}\left(\sum_{\left\{w, y \in w(X): w y=z, r_{w} \neq 0, s_{y} \neq 0\right\}} r_{w} s_{y}\right) z .
$$

This algebra is the free $R$-algebra on $X$ :

Proposition 2.6. The elements of $X$ generate $\mathbb{F}_{R}(w(X))$ as an $R$-algebra. Suppose that $f$ is a function from $X$ into an $R$-algebra $A$. Then there is an $R$-algebra homomorphism $\phi_{f}: \mathbb{F}_{R}(w(X)) \rightarrow A$ such that

$$
\phi_{f}\left(\sum_{w \in w(X)} r_{w} w\right)=\sum_{r_{w} \neq 0} r_{w} f\left(w_{1}\right) f\left(w_{2}\right) \cdots f\left(w_{|w|}\right) .
$$

Proof. Since each word $w$ is a product of $\left\{w_{i}: 1 \leq i \leq|w|\right\}$ and each $w_{i} \in X$, it is clear that $X$ generates $\mathbb{F}_{R}(w(X))$ as an algebra. We can extend $f$ to a function on $w(X)$ by setting $f(w)=f\left(w_{1}\right) f\left(w_{2}\right) \cdots f\left(w_{|w|}\right)$. Then the universal property of the free module $\mathbb{F}_{R}(w(X))$ gives a well-defined $R$-module homomorphism $\phi_{f}$ : $\mathbb{F}_{R}(w(X)) \rightarrow A$ satisfying (2.3). Now a straightforward calculation using (2.2) shows that $\phi_{f}$ is an $R$-algebra homomorphism.

We will want to put gradings on our free $R$-algebras, and the next proposition tells us how to do this.

Proposition 2.7. Suppose that $X$ is a nonempty set and $d$ is a function from $X$ to an additive abelian group $G$. Then there is a $G$-grading on $\mathbb{F}_{R}(w(X))$ such that

$$
\mathbb{F}_{R}(w(X))_{g}=\left\{\sum_{w \in w(X)} r_{w} w: r_{w} \neq 0 \Longrightarrow d(w):=\sum_{i=1}^{|w|} d\left(w_{i}\right)=g\right\} \quad \text { for } g \in G \text {. }
$$

Proof. It is straightforward that each $\mathbb{F}_{R}(w(X))_{g}$ is an additive subgroup of $\mathbb{F}_{R}(w(X))$. To see that they span, consider $a=\sum_{w \in w(X)} r_{w} w \in \mathbb{F}_{R}(w(X))$, and let $H:=\left\{w: r_{w} \neq 0\right\}$. For $g \in G$ and $w \in w(X)$, we define

$$
s_{g, w}= \begin{cases}r_{w} & \text { if } d(w)=g \\ 0 & \text { otherwise }\end{cases}
$$


then $a_{g}:=\sum_{w \in w(X)} s_{g, w} w$ belongs to $\mathbb{F}_{R}(w(X))_{g}$, and $\sum_{g \in d(H)} a_{g}$ is a finite sum which is easily seen to be $a$. To show that the $\mathbb{F}_{R}(w(X))_{g}$ are independent, suppose that $F$ is a finite subset of $G, a_{g} \in \mathbb{F}_{R}(w(X))_{g}$ and $\sum_{g \in F} a_{g}=0$. Write $a_{g}=$ $\sum_{w \in w(X)} t_{g, w} w$. Then $t_{g, w}=0$ unless $g=d(w)$, and

$$
0=\sum_{g \in F} \sum_{w \in w(X)} t_{g, w} w=\sum_{w \in w(X)}\left(\sum_{g \in F} t_{g, w}\right) w=\sum_{w \in w(X)} t_{d(w), w} w .
$$

Then, since the 0 element of $\mathbb{F}_{R}(X)$ is the sum in which all coefficients are 0 , we get $t_{d(w), w}=0$ for $w \in w(X)$. Thus we have $t_{g, w}=0$ for all $g, w$, and $a_{g}=0$ for all $g \in F$.

To see that $\mathbb{F}_{R}(w(X))_{g} \mathbb{F}_{R}(w(X))_{h} \subset \mathbb{F}_{R}(w(X))_{g+h}$, we take $\sum_{w \in w(X)} r_{w} w$ in $\mathbb{F}_{R}(w(X))_{g}$ and $\sum_{y \in w(X)} s_{y} y$ in $\mathbb{F}_{R}(w(X))_{h}$, and multiply them using (2.2). Suppose that the coefficient of $z$ on the right-hand side of (2.2) is nonzero. Then at least one summand $r_{w} s_{y}$ is nonzero, and for this summand $r_{w} \neq 0$ and $s_{y} \neq 0$, which by definition of the $\mathbb{F}_{R}(w(X))_{g}$ imply $d(w)=g$ and $d(y)=h$. But now $d(z)=d(w y)=d(w)+d(y)=g+h$, so the product is in $\mathbb{F}_{R}(w(X))_{g+h}$.

\section{KumJiAn-PASK FAMILIES}

The algebras of interest to us are algebraic analogues of a family of $C^{*}$-algebras introduced by Kumjian and Pask in 21. In the algebraic analogue, the generating relations look a little different, so we begin by examining algebraic consequences of the relations in [21. For the benefit of algebraists, we recall that a projection in a $C^{*}$-algebra $A$ is an element $P \in A$ such that $P^{*}=P=P^{2}$. A partial isometry is an element $S \in A$ such that $S=S S^{*} S$; equivalently, one of $S S^{*}$ or $S^{*} S$ is a projection, and then both are (see the appendix in [28], for example).

Let $\Lambda$ be a row-finite $k$-graph without sources. Kumjian and Pask studied collections $S=\left\{S_{\lambda}: \lambda \in \Lambda\right\}$ of partial isometries in a $C^{*}$-algebra $A$ such that

(a) $\left\{S_{v}: v \in \Lambda^{0}\right\}$ is a collection of mutually orthogonal projections,

(b) $S_{\lambda} S_{\mu}=S_{\lambda \mu}$ for $\lambda, \mu \in \Lambda$ with $r(\mu)=s(\lambda)$,

(c) $S_{\lambda}^{*} S_{\lambda}=S_{s(\lambda)}$ for $\lambda \in \Lambda$, and

(d) $S_{v}=\sum_{\lambda \in v \Lambda^{n}} S_{\lambda} S_{\lambda}^{*}$ for $v \in \Lambda^{0}$ and $n \in \mathbb{N}^{k}$.

Although they did not use this name, these quickly became known as Cuntz-Krieger $\Lambda$-families.

The relation (ㄷ) immediately implies that $S_{\lambda}=S_{\lambda}\left(S_{\lambda}^{*} S_{\lambda}\right)=S_{\lambda} S_{s(\lambda)}$. Next, recall that a finite sum $P=\sum_{i} P_{i}$ of projections in a $C^{*}$-algebra is a projection if and only if $P_{i} P_{j}=0$ for $i \neq j$, and then $P P_{i}=P_{i}$ for all $i$ (see [28, Corollary A.3]). Thus, since $S_{v}$ is a projection, relation (d) implies that if $\lambda, \mu \in v \Lambda^{n}$ and $\lambda \neq \mu$, then $\left(S_{\lambda} S_{\lambda}^{*}\right)\left(S_{\mu} S_{\mu}^{*}\right)=0$ and $S_{v}\left(S_{\lambda} S_{\lambda}^{*}\right)=\left(S_{\lambda} S_{\lambda}^{*}\right)$. In particular, we have $S_{r(\lambda)} S_{\lambda}=$ $S_{r(\lambda)}\left(S_{\lambda} S_{\lambda}^{*}\right) S_{\lambda}=\left(S_{\lambda} S_{\lambda}^{*}\right) S_{\lambda}=S_{\lambda}$. Next, note that

$$
S_{\lambda}^{*} S_{\mu}=S_{\lambda}^{*}\left(S_{\lambda} S_{\lambda}^{*}\right)\left(S_{\mu} S_{\mu}^{*}\right) S_{\mu},
$$

and hence we have the following stronger version of relation (ㄷ):

(ㄷ) if $\lambda, \mu \in v \Lambda^{n}$, then $S_{\lambda}^{*} S_{\mu}=\delta_{\lambda, \mu} S_{s(\lambda)}$.

The arguments in the previous paragraph do not work in the purely algebraic setting, and, as was the case for directed graphs in [1], we have to add some extra relations. 
If $\Lambda$ is a $k$-graph, we let $\Lambda^{\neq 0}:=\{\lambda \in \Lambda: d(\lambda) \neq 0\}$, and for each $\lambda \in \Lambda^{\neq 0}$ we introduce a ghost path $\lambda^{*}$; for $v \in \Lambda^{0}$, we define $v^{*}:=v$. We write $G(\Lambda)$ for the set of ghost paths, or $G\left(\Lambda^{\neq 0}\right)$ if we wish to exclude vertices. We define $d, r$ and $s$ on $G(\Lambda)$ by

$$
d\left(\lambda^{*}\right)=-d(\lambda), \quad r\left(\lambda^{*}\right)=s(\lambda), \quad s\left(\lambda^{*}\right)=r(\lambda)
$$

we then define composition on $G(\Lambda)$ by setting $\lambda^{*} \mu^{*}=(\mu \lambda)^{*}$ for $\lambda, \mu \in \Lambda^{\neq 0}$ with $r\left(\mu^{*}\right)=s\left(\lambda^{*}\right)$. The factorization property of $\Lambda$ induces a similar factorization property on $G(\Lambda)$.

Definition 3.1. Let $\Lambda$ be a row-finite $k$-graph without sources and let $R$ be a commutative ring with 1. A Kumjian-Pask $\Lambda$-family $(P, S)$ in an $R$-algebra $A$ consists of two functions $P: \Lambda^{0} \rightarrow A$ and $S: \Lambda^{\neq 0} \cup G\left(\Lambda^{\neq 0}\right) \rightarrow A$ such that:

(KP1) $\left\{P_{v}: v \in \Lambda^{0}\right\}$ is a family of mutually orthogonal idempotents,

(KP2) for all $\lambda, \mu \in \Lambda^{\neq 0}$ with $r(\mu)=s(\lambda)$, we have

$S_{\lambda} S_{\mu}=S_{\lambda \mu}, S_{\mu^{*}} S_{\lambda^{*}}=S_{(\lambda \mu)^{*}}, P_{r(\lambda)} S_{\lambda}=S_{\lambda}=S_{\lambda} P_{s(\lambda)}, P_{s(\lambda)} S_{\lambda^{*}}=S_{\lambda^{*}}=S_{\lambda^{*}} P_{r(\lambda)}$,

(KP3) for all $\lambda, \mu \in \Lambda^{\neq 0}$ with $d(\lambda)=d(\mu)$, we have

$$
S_{\lambda *} S_{\mu}=\delta_{\lambda, \mu} P_{s(\lambda)},
$$

(KP4) for all $v \in \Lambda^{0}$ and all $n \in \mathbb{N}^{k} \backslash\{0\}$, we have

$$
P_{v}=\sum_{\lambda \in v \Lambda^{n}} S_{\lambda} S_{\lambda^{*}}
$$

Remarks 3.2. (a) We have been careful to distinguish the vertex idempotents because we wanted to emphasise that there is only one generator for each path of degree 0 , whereas there are two for each path of nonzero degree. However, it is convenient when writing formulas such as (3.3) below to allow $S_{v}:=P_{v}$ and $S_{v^{*}}:=P_{v}$, and we do this.

(b) With the conventions we have set up, the last two relations in (KP2) can be summarized as $P_{r(x)} S_{x}=S_{x}=S_{x} P_{s(x)}$ for all $x \in \Lambda \cup G(\Lambda)$. This observation will be useful in calculations.

(c) Relations (KP2) and (KP3) imply that

$$
\left(S_{\lambda} S_{\lambda^{*}}\right)\left(S_{\lambda} S_{\lambda^{*}}\right)=S_{\lambda}\left(S_{\lambda^{*}} S_{\lambda}\right) S_{\lambda^{*}}=S_{\lambda} P_{s(\lambda)} S_{\lambda^{*}}=S_{\lambda} S_{\lambda^{*}},
$$

and (KP3) gives $\left(S_{\lambda} S_{\lambda^{*}}\right)\left(S_{\mu} S_{\mu^{*}}\right)=0$ when $d(\lambda)=d(\mu)$ and $\lambda \neq \mu$. Thus for each $n,\left\{S_{\lambda} S_{\lambda^{*}}: \lambda \in \Lambda^{n}\right\}$ is a set of mutually orthogonal idempotents.

The following analogue of [21, Lemma 3.1] tells us how to simplify products $S_{\lambda *} S_{\mu}$.

Lemma 3.3. Suppose that $(P, S)$ is a Kumjian-Pask $\Lambda$-family, and $\lambda, \mu \in \Lambda$. Then for each $q \geq d(\lambda) \vee d(\mu)$, we have

$$
S_{\lambda^{*}} S_{\mu}=\sum_{d(\lambda \alpha)=q, \lambda \alpha=\mu \beta} S_{\alpha} S_{\beta^{*}}
$$

Proof. By (KP2), we have $S_{\lambda^{*}} S_{\mu}=P_{s(\lambda)} S_{\lambda^{*}} S_{\mu} P_{s(\mu)}$, and then applying (KP4) at $v=s(\lambda)$ and at $v=s(\mu)$ gives

$$
S_{\lambda^{*}} S_{\mu}=\sum_{\alpha \in s(\lambda) \Lambda^{q-d(\lambda)}, \beta \in s(\mu) \Lambda^{q-d(\mu)}} S_{\alpha} S_{\alpha^{*}} S_{\lambda^{*}} S_{\mu} S_{\beta} S_{\beta^{*}} .
$$


Since $d(\lambda \alpha)=q=d(\mu \beta),(\mathrm{KP} 2)$ and (KP3) give

$$
S_{\alpha} S_{\alpha^{*}} S_{\lambda^{*}} S_{\mu} S_{\beta} S_{\beta^{*}}=S_{\alpha} S_{(\lambda \alpha)^{*}} S_{\mu \beta} S_{\beta^{*}}= \begin{cases}S_{\alpha} S_{\beta^{*}} & \text { if } \lambda \alpha=\mu \beta, \\ 0 & \text { otherwise, }\end{cases}
$$

and so the right-hand side of (3.1) collapses as required.

Theorem 3.4. Let $\Lambda$ be a row-finite k-graph without sources, and let $R$ be a commutative ring with 1 . Then there is an $R$-algebra $\operatorname{KP}_{R}(\Lambda)$ generated by a KumjianPask $\Lambda$-family $(p, s)$ such that, whenever $(Q, T)$ is a Kumjian-Pask $\Lambda$-family in an $R$-algebra $A$, there is a unique $R$-algebra homomorphism $\pi_{Q, T}: \operatorname{KP}_{R}(\Lambda) \rightarrow A$ such that

$$
\pi_{Q, T}\left(p_{v}\right)=Q_{v}, \quad \pi_{Q, T}\left(s_{\lambda}\right)=T_{\lambda}, \quad \pi_{Q, T}\left(s_{\mu^{*}}\right)=T_{\mu^{*}}
$$

for $v \in \Lambda^{0}$ and $\lambda, \mu \in \Lambda^{\neq 0}$. There is a $\mathbb{Z}^{k}$-grading on $\operatorname{KP}_{R}(\Lambda)$ satisfying

$$
\mathrm{KP}_{R}(\Lambda)_{n}=\operatorname{span}_{R}\left\{s_{\lambda} s_{\mu^{*}}: \lambda, \mu \in \Lambda \text { and } d(\lambda)-d(\mu)=n\right\},
$$

and we have $r p_{v} \neq 0$ for $v \in \Lambda^{0}$ and $r \in R \backslash\{0\}$.

Standard arguments show that $\left(\operatorname{KP}_{R}(\Lambda),(p, s)\right)$ is unique up to isomorphism, and we call $\operatorname{KP}_{R}(\Lambda)$ the Kumjian-Pask algebra of $\Lambda$ and $(p, s)$ the universal KumjianPask $\Lambda$-family.

Notation. We find it helpful to use the convention that lower-case letters signify that a Kumjian-Pask family $(p, s)$ has a universal property.

The proof of this theorem will occupy the rest of the section.

We begin by considering the free algebra $\mathbb{F}_{R}(w(X))$ on $X:=\Lambda^{0} \cup \Lambda^{\neq 0} \cup G\left(\Lambda^{\neq 0}\right)$. Let $I$ be the ideal of $\mathbb{F}_{R}(w(X))$ generated by the union of the following sets:

- $\left\{v w-\delta_{v, w} v: v, w \in \Lambda^{0}\right\}$;

- $\left\{\lambda-\mu \nu, \lambda^{*}-\nu^{*} \mu^{*}: \lambda, \mu, \nu \in \Lambda^{\neq 0}\right.$ and $\left.\lambda=\mu \nu\right\}$

$$
\cup\left\{r(\lambda) \lambda-\lambda, \lambda-\lambda s(\lambda), s(\lambda) \lambda^{*}-\lambda^{*}, \lambda^{*}-\lambda^{*} r(\lambda): \lambda \in \Lambda^{\neq 0}\right\} ;
$$

- $\left\{\lambda^{*} \mu-\delta_{\lambda, \mu} s(\lambda): \lambda, \mu \in \Lambda^{\neq 0}\right.$ such that $\left.d(\lambda)=d(\mu)\right\}$;

- $\left\{v-\sum_{\lambda \in v \Lambda^{n}} \lambda \lambda^{*}: v \in \Lambda^{0}, n \in \mathbb{N}^{k} \backslash\{0\}\right\}$.

We now define $\operatorname{KP}_{R}(\Lambda):=\mathbb{F}_{R}(w(X)) / I$. Let $q: \mathbb{F}_{R}(w(X)) \rightarrow \mathbb{F}_{R}(w(X)) / I$ be the quotient map. Then $\left\{p_{v}, s_{\lambda}, s_{\mu^{*}}\right\}:=\left\{q(v), q(\lambda), q\left(\mu^{*}\right)\right\}$ gives a generating KumjianPask $\Lambda$-family $(p, s)$ in $\operatorname{KP}_{R}(\Lambda)$.

Now let $(Q, T)$ be a Kumjian-Pask $\Lambda$-family in an $R$-algebra $A$. Define $f_{Q, T}$ : $X \rightarrow A$ by $f(v)=Q_{v}, f(\lambda)=T_{\lambda}$ and $f\left(\mu^{*}\right)=T_{\mu^{*}}$, and the universal property of $\mathbb{F}_{R}(w(X))$ described in Proposition 2.6 gives an $R$-algebra homomorphism $\phi_{f}$ : $\mathbb{F}_{R}(w(X)) \rightarrow A$ such that $\phi_{f}(v)=Q_{v}, \phi_{f}(\lambda)=T_{\lambda}$ and $\phi_{f}\left(\mu^{*}\right)=T_{\mu^{*}}$. The Kumjian-Pask relations imply that $\phi_{f}$ vanishes on the ideal $I$, and therefore factors through an $R$-algebra homomorphism $\pi_{Q, T}: \mathrm{KP}_{R}(\Lambda) \rightarrow A$ satisfying (3.2). Since the elements in $X$ generate $\mathbb{F}_{R}(w(X))$ as an algebra, there is exactly one such homomorphism.

Applying Proposition 2.7 to the degree map $d: X \rightarrow \mathbb{N}^{k}$ gives a $\mathbb{Z}^{k}$-grading of the free algebra $\mathbb{F}_{R}(w(X))$, and every generator of $I$ lies in one of the subgroups $\mathbb{F}_{R}(w(X))_{n}$ of homogeneous elements. Thus the ideal $I$ is graded, and the quotient $\mathrm{KP}_{R}(\Lambda)=\mathbb{F}_{R}(w(X)) / I$ is graded by the subgroups $q\left(\mathbb{F}_{R}(w(X))_{n}\right)$. The following lemma identifies $q\left(\mathbb{F}_{R}(w(X))_{n}\right)$ with the subgroup $\operatorname{KP}_{R}(\Lambda)_{n}$ described in (3.3). 
Lemma 3.5. For every $w \in w(X)$, we have $q(w) \in \mathrm{KP}_{R}(\Lambda)_{d(w)}$.

Proof. We will prove this by induction on $|w|$. For $|w|=0$ or 1 , the result is covered by the convention in Remark 3.2 (a) that we can view vertices as paths or ghost paths, and hence can add appropriate factors $s_{v}=p_{v}$ or $s_{v^{*}}=p_{v}$ without changing $q(w)$.

For $|w|=2$, there are four cases to consider: $w=\lambda \mu^{*}, w=\lambda^{*} \mu, w=\lambda \mu$, $w=\mu^{*} \lambda^{*}$. For the first, we have $q(w)=s_{\lambda} s_{\mu^{*}}$, and there is nothing to prove. For the second, we apply Lemma 3.3. and observe that $\lambda \alpha=\mu \beta$ implies $d(\alpha)-d(\beta)=$ $d(\mu)-d(\lambda)=d(w)$. For the third, we notice that the result is trivial if $q(w)=0$, and if not, (KP2) gives $0 \neq q(w)=s_{\lambda} p_{s(\lambda)} p_{r(\mu)} s_{\mu}$, which implies that $s(\lambda)=r(\mu)$ and that $s_{\lambda} s_{\mu}=s_{\lambda \mu} s_{s(\mu)^{*}}$ belongs to $\operatorname{KP}_{R}(\Lambda)_{d(w)}$. A similar argument works in the fourth case.

Now suppose that $n \geq 2$ and $q(y) \in \operatorname{KP}_{R}(\Lambda)_{d(y)}$ for every word $y$ with $|y| \leq n$. Let $w$ be a word with $|w|=n+1$ and $q(w) \neq 0$. If $w$ contains a subword $w_{i} w_{i+1}=$ $\lambda \mu$, then inserting vertex idempotents shows that $s(\lambda)=r(\mu)$, so that $\lambda$ and $\mu$ are composable in $\Lambda$. We now let $w^{\prime}$ be the word obtained from $w$ by replacing $w_{i} w_{i+1}$ with the single path $\lambda \mu$, and then

$$
q(w)=s_{w_{1}} \cdots s_{w_{i-1}} s_{\lambda} s_{\mu} s_{w_{i+2}} \cdots s_{w_{n+1}}=s_{w_{1}} \cdots s_{w_{i-1}} s_{\lambda \mu} s_{w_{i+2}} \cdots s_{w_{n+1}}=q\left(w^{\prime}\right) .
$$

Since $\left|w^{\prime}\right|=n$ and $d\left(w^{\prime}\right)=d(w)$, the inductive hypothesis implies that $q(w) \in$ $\mathrm{KP}_{R}(\Lambda)_{d(w)}$. A similar argument shows that $q(w) \in \mathrm{KP}_{R}(\Lambda)_{d(w)}$ whenever $w$ contains a subword $w_{i} w_{i+1}=\lambda^{*} \mu^{*}$.

If $w$ contains no subword of the form $\lambda \mu$ or $\lambda^{*} \mu^{*}$, then it must consist of alternating paths and ghost paths. In particular, remembering that $|w|=n+1 \geq 3$, we see that either $w_{1} w_{2}$ or $w_{2} w_{3}$ has the form $\lambda^{*} \mu$. Now we can use Lemma 3.3 to write $q(w)$ as a sum of terms $q\left(y^{i}\right)$ with $\left|y^{i}\right|=n+1$ and $d\left(y^{i}\right)=d(w)$. Each nonzero summand $q\left(y^{i}\right)$ contains a factor of the form $s_{\beta^{*}} s_{\gamma^{*}}$ or one of the form $s_{\delta} s_{\alpha}$, and the argument of the preceding paragraph shows that every $q\left(y^{i}\right) \in \operatorname{KP}_{R}(\Lambda)_{d(w)}$. Thus so is their sum $q(w)$.

It remains to prove that the elements $r p_{v}$ with $r \neq 0$ are nonzero, and for this it suffices to produce a Kumjian-Pask $\Lambda$-family $(Q, T)$ in an $R$-algebra such that each $r Q_{v}$ is nonzero. We do this by modifying the construction in [21, Proposition 2.11]. Let $\mathbb{F}_{R}\left(\Lambda^{\infty}\right)$ be the free module with basis the infinite path space. We next fix $v \in \Lambda^{0}$ and $\lambda, \mu \in \Lambda^{\neq 0}$, and use the composition and factorization constructions of Lemma 2.5 to define functions $f_{v}, f_{\lambda}, f_{\mu^{*}}: \Lambda^{\infty} \rightarrow \mathbb{F}_{R}\left(\Lambda^{\infty}\right)$ by

$$
\begin{aligned}
f_{v}(x) & = \begin{cases}x & \text { if } x(0)=v, \\
0 & \text { otherwise; }\end{cases} \\
f_{\lambda}(x) & = \begin{cases}\lambda x & \text { if } x(0)=s(\lambda), \\
0 & \text { otherwise; and }\end{cases} \\
f_{\mu^{*}}(x) & = \begin{cases}x(d(\mu), \infty) & \text { if } x(0, d(\mu))=\mu, \\
0 & \text { otherwise }\end{cases}
\end{aligned}
$$

The universal property of free modules now gives nonzero endomorphisms $Q_{v}, T_{\lambda}$, $T_{\mu^{*}}: \mathbb{F}_{R}\left(\Lambda^{\infty}\right) \rightarrow \mathbb{F}_{R}\left(\Lambda^{\infty}\right)$ extending $f_{v}, f_{\lambda}$ and $f_{\mu^{*}}$.

It is straightforward to check using Lemma 2.5 that $(Q, T)$ is a Kumjian-Pask $\Lambda$-family in $\operatorname{End}\left(\mathbb{F}_{R}\left(\Lambda^{\infty}\right)\right)$. For example, to verify (KP3), suppose that $d(\lambda)=d(\mu)$ 
and $x \in \Lambda^{\infty}$. Then

$$
\begin{aligned}
T_{\lambda^{*}} T_{\mu}(x) & = \begin{cases}T_{\lambda^{*}}(\mu x) & \text { if } x(0)=s(\mu), \\
0 & \text { otherwise }\end{cases} \\
& = \begin{cases}(\mu x)(d(\lambda), \infty) & \text { if } x(0)=s(\mu) \text { and }(\mu x)(0, d(\lambda))=\lambda, \\
0 & \text { otherwise. }\end{cases}
\end{aligned}
$$

Since $d(\lambda)=d(\mu)$, Lemma 2.5 implies that $(\mu x)(0, d(\lambda))=(\mu x)(0, d(\mu))=\mu$ if $r(x)=s(\mu)$, so $T_{\lambda *} T_{\mu}(x)$ vanishes for all $x$ unless $\lambda=\mu$, and then is $x$ if and only if $r(x)=s(\mu)$. But this is exactly what $Q_{s(\mu)}$ does to $x$, and hence we have $T_{\lambda^{*}} T_{\mu}=Q_{s(\mu)}$.

Since $(Q, T)$ is a Kumjian-Pask $\Lambda$-family, there exists an $R$-algebra homomorphism $\pi_{Q, T}: \operatorname{KP}_{R}(\Lambda) \rightarrow \operatorname{End}\left(\mathbb{F}_{R}\left(\Lambda^{\infty}\right)\right)$ such that $\pi_{Q, T}\left(p_{v}\right)=Q_{v}, \pi_{Q, T}\left(s_{\lambda}\right)=T_{\lambda}$ and $\pi_{Q, T}\left(s_{\mu^{*}}\right)=T_{\mu}$. Since every vertex $v$ is the range of an infinite path, if $r \neq 0$, then $r Q_{v} \neq 0$. It follows that $r p_{v} \neq 0$ too, and this completes the proof of Theorem 3.4

We call the $R$-algebra homomorphism $\pi_{Q, T}: \mathrm{KP}_{R}(\Lambda) \rightarrow \operatorname{End}\left(\mathbb{F}_{R}\left(\Lambda^{\infty}\right)\right)$ constructed above the infinite-path representation of $\mathrm{KP}_{R}(\Lambda)$.

\section{The UNIQUENESS THEOREMS}

Let $\Lambda$ be a row-finite $k$-graph without sources. We write $(p, s)$ for the universal Kumjian-Pask $\Lambda$-family in $\operatorname{KP}_{R}(\Lambda)$. In this section we prove graded-uniqueness and Cuntz-Krieger uniqueness theorems for $\mathrm{KP}_{R}(\Lambda)$.

Theorem 4.1 (The graded-uniqueness theorem). Let $\Lambda$ be a row-finite $k$-graph without sources, $R$ a commutative ring with 1 , and $A$ a $\mathbb{Z}^{k}$-graded ring. If $\pi$ : $\mathrm{KP}_{R}(\Lambda) \rightarrow A$ is a $\mathbb{Z}^{k}$-graded ring homomorphism such that $\pi\left(r p_{v}\right) \neq 0$ for all $r \in R \backslash\{0\}$ and $v \in \Lambda^{0}$, then $\pi$ is injective.

The next two lemmas are the first steps in the proofs of both uniqueness theorems.

Lemma 4.2. Every nonzero $x \in \operatorname{KP}_{R}(\Lambda)$ can be written as a sum $\sum_{(\alpha, \beta) \in F} r_{\alpha, \beta} s_{\alpha} s_{\beta^{*}}$, where $F$ is a finite subset of $\Lambda \times \Lambda, r_{\alpha, \beta} \in R \backslash\{0\}$ for all $(\alpha, \beta) \in F$, and all the $\beta$ have the same degree. In this case we say $x$ is written in normal form.

Proof. By Theorem 3.4, we can write $x$ as a finite sum $x=\sum_{(\sigma, \tau) \in G} r_{\sigma, \tau} s_{\sigma} s_{\tau^{*}}$ with each $r_{\sigma, \tau} \neq 0$. Set $m=\bigvee_{(\sigma, \tau) \in G} d(\tau)$. For each $(\sigma, \tau) \in G$, applying (KP4) with $n_{\tau}:=m-d(\tau)$ gives

$$
s_{\sigma} s_{\tau^{*}}=s_{\sigma} p_{s(\sigma)} s_{\tau^{*}}=\sum_{\lambda \in s(\sigma) \Lambda^{n_{\tau}}} s_{\sigma \lambda} s_{(\tau \lambda) *}
$$

substituting back into the expression for $x$ and combining terms gives the result.

Lemma 4.3. Suppose that $x$ is a nonzero element of $\operatorname{KP}_{R}(\Lambda)$ and $x=$ $\sum_{(\alpha, \beta) \in F} r_{\alpha, \beta} s_{\alpha} s_{\beta^{*}}$ is in normal form. Then there exists $\gamma \in F_{2}:=\{\beta:(\alpha, \beta) \in$ $F$ for some $\alpha \in \Lambda$ \} such that

$$
0 \neq x s_{\gamma}=\sum_{\alpha \in G} r_{\alpha, \gamma} s_{\alpha} \quad \text { where } G:=\{\alpha:(\alpha, \gamma) \in F\}
$$


Further, if $\delta \in G$, then

$$
0 \neq s_{\delta^{*}} x s_{\gamma}=r_{\delta, \gamma} p_{s(\delta)}+\sum_{\{\alpha \in G: \alpha \neq \delta\}} r_{\alpha, \gamma} s_{\delta^{*}} s_{\alpha},
$$

and $r_{\delta, \gamma} p_{s(\delta)}$ is the 0-graded component of $s_{\delta^{*}} x s_{\gamma}$.

Proof. Since all $\beta$ in $F_{2}$ have the same degree, (KP3) implies that $\left\{s_{\beta} s_{\beta^{*}}: \beta \in\right.$ $\left.F_{2}\right\}$ is a set of mutually orthogonal idempotents. Then $p=\sum_{\beta \in F_{2}} s_{\beta} s_{\beta^{*}}$ is an idempotent and satisfies $x p=x$. In particular, $x p \neq 0$, and hence there exists $\gamma \in F_{2}$ such that $x s_{\gamma} \neq 0$. Now (KP3) gives

$$
0 \neq x s_{\gamma}=\sum_{(\alpha, \beta) \in F} r_{\alpha, \beta} s_{\alpha} s_{\beta^{*}} s_{\gamma}=\sum_{\{(\alpha, \beta) \in F: \beta=\gamma\}} r_{\alpha, \beta} s_{\alpha}=\sum_{\alpha \in G} r_{\alpha, \gamma} s_{\alpha},
$$

and for $\delta \in G$, we have

$$
s_{\delta^{*}} x s_{\gamma}=\sum_{\alpha \in G} r_{\alpha, \gamma} s_{\delta^{*}} s_{\alpha}=r_{\delta, \gamma} p_{s(\delta)}+\sum_{\{\alpha \in G: \alpha \neq \delta\}} r_{\alpha, \gamma} s_{\delta^{*}} s_{\alpha} .
$$

If $s_{\delta^{*}} s_{\alpha} \neq 0$ and $\alpha \neq \delta$, then $d(\alpha) \neq d(\delta)$ by (KP3), and $s_{\delta^{*}} s_{\alpha}$ is a sum of monomials $s_{\mu} s_{\nu^{*}}$ all of which have degree $d(\mu)-d(\nu)=d(\alpha)-d(\delta) \neq 0$ (see Lemma 3.3). Thus $r_{\delta, \gamma} p_{s(\delta)}$ is the 0 -graded component of $s_{\delta^{*}} x s_{\gamma}$. Since $r_{\delta, \gamma} p_{s(\delta)} \neq 0$, we have $s_{\delta^{*}} x s_{\gamma} \neq 0$ too.

Proof of Theorem 4.1. Let $0 \neq x \in \mathrm{KP}_{R}(\Lambda)$. By Lemma 4.2, $x$ can be written in normal form, and by Lemma 4.3 there exist a finite set $G$ and $\gamma, \delta \in \Lambda$ such that (4.2) holds and $r_{\delta, \gamma} p_{s(\delta)}$ is the 0 -graded component of $s_{\delta^{*}} x s_{\gamma}$. Since $\pi$ is $\mathbb{Z}^{k}$ graded, $\pi\left(r_{\delta, \gamma} p_{s(\delta)}\right)$ is the 0 -graded component of $\pi\left(s_{\delta^{*}} x s_{\gamma}\right)$, and since $\pi\left(r_{\delta, \gamma} p_{s(\delta)}\right)$ is nonzero by assumption, so is $\pi\left(s_{\delta^{*}} x s_{\gamma}\right)$. Since $\pi$ is a ring homomorphism, we deduce that $\pi(x) \neq 0$, and hence that $\pi$ is injective.

Remark 4.4. The graded-uniqueness theorem is an analogue of the gauge-invariant uniqueness theorems for graph $C^{*}$-algebras, and we will discuss the relationship in 97.1. The first gauge-invariant uniqueness theorem was for Cuntz-Krieger algebras [19. Theorem 2.3]; the first versions for graph $C^{*}$-algebras and higher-rank graph algebras were [9, Theorem 2.1] and [21, Theorem 3.4]. The graded-uniqueness theorem for Leavitt path algebras was originally derived from the classification of the graded ideals; direct proofs were given in [29] and [37. Theorem 4.1] and its proof were motivated by [38, Theorem 6.5].

For the Cuntz-Krieger uniqueness theorem, we need an aperiodicity condition on $\Lambda$. Following Robertson and Sims [32, we say that a $k$-graph $\Lambda$ is aperiodic if for every $v \in \Lambda^{0}$ and $m \neq n \in \mathbb{N}^{k}$ there exists $\lambda \in v \Lambda$ such that $d(\lambda) \geq m \vee n$ and

$$
\lambda(m, m+d(\lambda)-(m \vee n)) \neq \lambda(n, n+d(\lambda)-(m \vee n)) .
$$

We say $\Lambda$ is periodic if $\Lambda$ is not aperiodic. Several aperiodicity conditions appear in the literature, but they are all equivalent when $\Lambda$ is row-finite without sources. We find the finite path formulation of aperiodicity from [32] easier to understand, and it allows us to borrow arguments from [17] which do not require readers to know about the different formulations in [21] and [30].

Example 4.5. Let $\Lambda$ be a row-finite 1-graph without sources, and let $E=$ $\left(E^{0}, E^{1}, r, s\right)$ be the associated directed graph. Then $\Lambda$ is aperiodic if and only 
if for every $v \in E^{0}$ and every $m, n \in \mathbb{N}$ with $m<n$, there exists a path $\lambda$ with $r(\lambda)=v,|\lambda| \geq n$ and $\lambda_{m+1} \ldots \lambda_{m+|\lambda|-n} \neq \lambda_{n+1} \ldots \lambda_{|\lambda|}$.

The following reassuring lemma tells us that, for a directed graph, aperiodicity is equivalent to the usual hypothesis of Cuntz-Krieger uniqueness theorems.

Lemma 4.6. Let $\Lambda$ be a 1-graph and $E$ its associated directed graph. Then $\Lambda$ is aperiodic if and only if every cycle in $E$ has an entry.

Proof. Suppose that $E$ has a cycle $\mu$ of length $k \geq 1$ without an entry, and take $v=r(\mu), m=0$ and $n=k$. Since $\mu$ has no entry, the only paths $\lambda$ with $r(\lambda)=$ $r(\mu)$ and length at least $k$ have the form $\mu^{l} \mu^{\prime}$, where $l \geq 1$ and $\mu=\mu^{\prime} \mu^{\prime \prime}$; then $\lambda_{1} \cdots \lambda_{|\lambda|-k}=\mu^{l-1} \mu^{\prime}=\lambda_{k+1} \cdots \lambda_{|\lambda|}$ for every such $\lambda$, which shows that $\Lambda$ is periodic.

Conversely, suppose that every cycle in $E$ has an entry. Fix $v \in E^{0}$ and $m<n$ in $\mathbb{N}$. First, suppose that $v$ can be reached from a cycle $\mu$, that is, there exists $\alpha$ with $r(\alpha)=v$ such that $\alpha \mu$ is a path. Then $\mu$ has an entry $e \in E^{1}$, and we may suppose by adjusting $\alpha$ that $s(\mu)=r(e)$. Now choose a path of the form $\lambda=\alpha \mu \mu \ldots \mu e$ such that $\lambda_{m}$ is an edge in $\mu$ and $|\lambda| \geq n$. Then $\lambda_{m+|\lambda|-n} \neq \lambda_{|\lambda|}$. Second, suppose that $v$ cannot be reached from a cycle. Choose $\lambda$ with $r(\lambda)=v$ and $|\lambda|>n$. Then $\lambda_{m+1} \ldots \lambda_{m+|\lambda|-n} \neq \lambda_{n+1} \ldots \lambda_{|\lambda|}$ because otherwise $\lambda_{m+1} \ldots \lambda_{n}$ would be a return path which connects to $v$, and which would contain a cycle connecting to $v$. So either way, the aperiodicity condition holds for $m, n$ and $v$, and $\Lambda$ is aperiodic.

We can now state our second uniqueness theorem.

Theorem 4.7 (The Cuntz-Krieger uniqueness theorem). Let $\Lambda$ be an aperiodic row-finite $k$-graph without sources, let $R$ be a commutative ring with 1 , and let $A$ be a ring. If $\pi: \mathrm{KP}_{R}(\Lambda) \rightarrow A$ is a ring homomorphism such that $\pi\left(r p_{v}\right) \neq 0$ for all $r \in R \backslash\{0\}$ and $v \in \Lambda^{0}$, then $\pi$ is injective.

We need two preliminary results for the proof. Lemma 4.8 was an ingredient in the proof of the $C^{*}$-algebraic uniqueness theorem in [17, and Proposition 4.9] will be needed again in our analysis of the ideal structure in 6 .

Lemma 4.8 ([17, Lemma 6.2]). Suppose that $\Lambda$ is an aperiodic row-finite $k$-graph without sources, and fix $v \in \Lambda^{0}$ and $m \in \mathbb{N}^{k}$. Then there exists $\lambda \in \Lambda$ with $r(\lambda)=v$ and $d(\lambda) \geq m$ such that

$$
\begin{aligned}
& \left.\begin{array}{l}
\alpha, \beta \in \Lambda, s(\alpha)=s(\beta)=v, d(\alpha), d(\beta) \leq m, \\
\text { and }(\alpha \lambda)(0, d(\lambda))=(\beta \lambda)(0, d(\lambda))
\end{array}\right\} \Longrightarrow \alpha=\beta .
\end{aligned}
$$

Proposition 4.9. Let $\Lambda$ be an aperiodic row-finite $k$-graph without sources and let $R$ be a commutative ring with 1 . Let $x=\sum_{(\alpha, \beta) \in F} r_{\alpha, \beta} s_{\alpha} s_{\beta^{*}}$ be a nonzero element of $\operatorname{KP}_{R}(\Lambda)$ in normal form. Then there exist $\sigma, \tau \in \Lambda,(\delta, \gamma) \in F$ and $w \in \Lambda^{0}$ such that $s_{\sigma^{*}} x s_{\tau}=r_{\delta, \gamma} p_{w}$.

Proof. Lemma 4.3 implies that there exists $\gamma \in \Lambda$ such that $G:=\{\alpha:(\alpha, \gamma) \in F\}$ is nonempty and

$$
0 \neq s_{\delta^{*}} x s_{\gamma}=r_{\delta, \gamma} p_{s(\delta)}+\sum_{\{\alpha \in G: \alpha \neq \delta\}} r_{\alpha, \gamma} s_{\delta^{*}} s_{\alpha} \quad \text { for every } \delta \in G .
$$


Since $\Lambda$ is aperiodic we can apply Lemma 4.8 with $v=s(\delta)$ and $m=\bigvee_{\alpha \in G} d(\alpha)$ to find $\lambda \in s(\delta) \Lambda$ with $d(\lambda) \geq m$ such that (4.4) holds. Now

$$
s_{\lambda^{*}}\left(s_{\delta^{*}} x s_{\gamma}\right) s_{\lambda}=r_{\delta, \gamma} p_{s(\lambda)}+\sum_{\{\alpha \in G: \alpha \neq \delta\}} r_{\alpha, \gamma} s_{(\delta \lambda)^{*}} s_{\alpha \lambda} .
$$

If the summand $s_{(\delta \lambda) *} s_{\alpha \lambda}$ is nonzero, then $s_{(\delta \lambda)(0, d(\lambda))^{*}} s_{(\alpha \lambda)(0, d(\lambda))}$ is nonzero, (KP3) implies that $(\delta \lambda)(0, d(\lambda))=(\alpha \lambda)(0, d(\lambda))$, and (4.4) implies that $\alpha=\delta$. Thus (4.5) collapses to $s_{(\delta \lambda) *} x s_{\gamma \lambda}=r_{\delta, \gamma} p_{s(\lambda)}$, and we can take $\sigma=\delta \lambda$ and $\tau=\gamma \lambda$.

Proof of Theorem 4.7. Let $0 \neq x \in \mathrm{KP}_{R}(\Lambda)$. By Lemma 4.2 we can write $x$ in normal form. By Proposition 4.9 there exist $\sigma, \tau \in \Lambda$ and $r \in R \backslash\{0\}$ such that $s_{\sigma^{*}} x s_{\tau}=r p_{w}$ for some $w \in \Lambda^{0}$. Now

$$
\pi\left(s_{\sigma^{*}}\right) \pi(x) \pi\left(s_{\tau}\right)=\pi\left(s_{\sigma^{*}} x s_{\tau}\right)=\pi\left(r p_{w}\right) \neq 0
$$

by assumption, and so $\pi(x) \neq 0$. Thus $\pi$ is injective.

The Cuntz-Krieger uniqueness theorem immediately gives:

Corollary 4.10. Let $\Lambda$ be an aperiodic row-finite $k$-graph without sources. Then the infinite-path representation $\pi_{Q, T}: \mathrm{KP}_{R}(\Lambda) \rightarrow \operatorname{End}\left(\mathbb{F}_{R}\left(\Lambda^{\infty}\right)\right)$ from the end of $\S 3$ is injective.

We will see in Lemma 5.9 below that $\pi_{Q, T}$ is not injective when $\Lambda$ is periodic.

Remark 4.11. The uniqueness theorem for Cuntz-Krieger algebras was proved in [12, and extended to graph algebras in [22] and higher-rank graph algebras in 21. The first versions for Leavitt algebras were in [1, 29, 37. All require some form of aperiodicity condition. For graphs, everybody now uses the condition (L) from [22], which says that every cycle has an entry. For row-finite higher-rank graphs without sources, all the formulations are equivalent to the finite-path formulation which we use here [32, Lemma 3.2]. When there are sources or infinite receivers, one has to be a bit more careful, and we refer to [24] for a detailed discussion.

\section{BASIC IDEALS AND BASIC SIMPLICITY}

Let $\Lambda$ be a row-finite $k$-graph without sources; we continue to write $(p, s)$ for the universal Kumjian-Pask $\Lambda$-family in $\mathrm{KP}_{R}(\Lambda)$.

A subset $H$ of $\Lambda^{0}$ is hereditary if $\lambda \in \Lambda$ and $r(\lambda) \in H$ imply $s(\lambda) \in H$. A subset $H$ is saturated if $v \in \Lambda^{0}, n \in \mathbb{N}^{k}$ and $s\left(v \Lambda^{n}\right) \subset H$ imply $v \in H$. For a saturated hereditary subset $H$, we write $I_{H}$ for the ideal of $\operatorname{KP}_{R}(\Lambda)$ generated by $\left\{p_{v}: v \in H\right\}$.

The standard path for studying graph algebras predicts that $H \mapsto I_{H}$ should be a bijection between the saturated hereditary subsets of $\Lambda^{0}$ and the graded ideals of $\operatorname{KP}_{R}(\Lambda)$. However, since we are allowing coefficients in a commutative ring, we have to follow 38 and restrict attention to the basic ideals, which are the ideals $I$ such that $r p_{v} \in I$ and $r \in R \backslash\{0\}$ imply $p_{v} \in I$. This assumption gets us back on path:

Theorem 5.1. Let $\Lambda$ be a row-finite $k$-graph without sources and let $R$ be a commutative ring with 1 . Then the map $H \mapsto I_{H}$ is a lattice isomorphism from the lattice of saturated hereditary subsets of $\Lambda^{0}$ onto the lattice of basic and graded ideals of $\mathrm{KP}_{R}(\Lambda)$. 
The proof of Theorem 5.1 follows the general path first taken in $9, \S 4]$. The first lemma is a little more general than we need right now, but the sets $H_{I, r}$ will be of interest in 86 .

Lemma 5.2. Let $I$ be an ideal of $\operatorname{KP}_{R}(\Lambda)$ and $r \in R$. Then $H_{I, r}:=\left\{v \in \Lambda^{0}\right.$ : $\left.r p_{v} \in I\right\}$ is a saturated hereditary subset of $\Lambda^{0}$. In particular, $H_{I}:=H_{I, 1}=\{v \in$ $\left.\Lambda^{0}: p_{v} \in I\right\}$ is saturated and hereditary.

Proof. To see that $H_{I, r}$ is hereditary, suppose $\lambda \in \Lambda$ and $r(\lambda) \in H_{I, r}$. Then $r p_{r(\lambda)} \in I$ and $r s_{\lambda}=r p_{r(\lambda)} s_{\lambda} \in I$. Now $r p_{s(\lambda)}=r s_{\lambda^{*}} s_{\lambda}=s_{\lambda^{*}} r s_{\lambda} \in I$. Thus $s(\lambda) \in H_{I, r}$, and $H_{I, r}$ is hereditary. To see that $H_{I, r}$ is saturated, fix $v \in \Lambda^{0}$ and $n \in \mathbb{N}^{k}$, and suppose that $s(\lambda) \in H_{I, r}$ for all $\lambda \in v \Lambda^{n}$. Then $r p_{s(\lambda)} \in I$ for all $\lambda \in v \Lambda^{n}$, and (KP4) gives

$$
r p_{v}=\sum_{\lambda \in v \Lambda^{n}} r s_{\lambda} s_{\lambda^{*}}=\sum_{\lambda \in v \Lambda^{n}} s_{\lambda}\left(r p_{s(\lambda)}\right) s_{\lambda^{*}} \in I .
$$

Thus $v \in H_{I, r}$, and $H_{I, r}$ is saturated.

Lemma 5.3. Suppose $\Lambda$ is a row-finite $k$-graph without sources and $H$ is a saturated hereditary subset of $\Lambda^{0}$. Then $\Lambda \backslash H:=\left(\Lambda^{0} \backslash H, s^{-1}\left(\Lambda^{0} \backslash H\right), r, s\right)$ is a row-finite $k$-graph without sources, and if $(Q, T)$ is a Kumjian-Pask family for $\Lambda \backslash H$ in an $R$-algebra $A$, then

$$
P_{v}=\left\{\begin{array}{ll}
Q_{v} & \text { if } v \notin H \\
0 & \text { otherwise, }
\end{array} \quad S_{\lambda}=\left\{\begin{array}{ll}
T_{\lambda} & \text { if } s(\lambda) \notin H \\
0 & \text { otherwise, }
\end{array} \quad \text { and } \quad S_{\mu^{*}}= \begin{cases}T_{\mu^{*}} & \text { if } s(\mu) \notin H \\
0 & \text { otherwise }\end{cases}\right.\right.
$$

form a Kumjian-Pask $\Lambda$-family $(P, S)$ in $A$.

Proof. It is straightforward to check that $\Lambda \backslash H$ is a subcategory of $\Lambda$, and the hereditariness of $H$ implies that if $\lambda \in \Lambda \backslash H$ and $\lambda=\mu \nu$, then the factors $\mu$ and $\nu$ have source in $\Lambda^{0} \backslash H$ (see [30, Theorem 5.2(b)]). So $\Lambda \backslash H$ is a row-finite $k$-graph. To see that $\Lambda \backslash H$ has no sources, suppose that $v \in(\Lambda \backslash H)^{0}=\Lambda^{0} \backslash H$ and $n \in \mathbb{N}^{k}$. Since $\Lambda$ has no sources, $v \Lambda^{n}$ is nonempty, and if $s(\lambda) \in H$ for every $\lambda \in v \Lambda^{n}$, then $v \in H$ because $H$ is saturated, which contradicts $v \in \Lambda^{0} \backslash H$. Thus there must exist $\lambda \in v \Lambda^{n}$ such that $s(\lambda) \in \Lambda^{0} \backslash H$, and then $\lambda \in v(\Lambda \backslash H)^{n}$, so $v$ is not a source in $\Lambda \backslash H$.

Most of the Kumjian-Pask relations $(\mathrm{KP} 1-3)$ for $(P, S)$ follow immediately from those for $(Q, T)$, though we have to use that $H$ is hereditary to see that $s(\lambda) \notin H$ implies $r(\lambda) \notin H$, so that $S_{\lambda}=T_{\lambda}=Q_{r(\lambda)} T_{\lambda}=P_{r(\lambda)} S_{\lambda}$ in (KP2). For (KP4), we observe that the nonzero terms in $\sum_{\lambda \in v \Lambda^{n}} S_{\lambda} S_{\lambda^{*}}$ are parametrized by

$$
\left\{\lambda \in v \Lambda^{n}: s(\lambda) \notin H\right\}= \begin{cases}\emptyset & \text { if } v \in H, \\ v(\Lambda \backslash H)^{n} & \text { if } v \notin H .\end{cases}
$$

Recall that an ideal $I$ is idempotent if $I=I^{2}$ in the sense that $I$ is spanned by products $a b$ with $a, b \in I$.

Lemma 5.4. Let $H$ be a saturated hereditary subset of $\Lambda^{0}$. Then

$$
I_{H}=\operatorname{span}_{R}\left\{s_{\sigma} s_{\lambda^{*}}: s(\sigma)=s(\lambda) \in H\right\},
$$

$I_{H}$ is a basic, graded and idempotent ideal of $\mathrm{KP}_{R}(\Lambda)$, and $H_{I_{H}}=H$. 
Proof. Since $s_{\sigma} s_{\lambda^{*}}=s_{\sigma} p_{s(\sigma)} s_{\lambda^{*}}$, the right-hand side $J$ of (5.1) is contained in $I_{H}$, and it contains all the generators $p_{v}$ (by the convention in Remark 3.2). So to prove (5.1), it suffices for us to prove that $J$ is an ideal. To see this, consider $s_{\sigma} s_{\lambda^{*}}$ with $s(\sigma)=s(\lambda) \in H$ and $s_{\mu} s_{\delta^{*}} \in \mathrm{KP}_{R}(\Lambda)$. Applying Lemma 3.3 with $q=d(\lambda) \vee d(\mu)$ gives

$$
s_{\sigma} s_{\lambda^{*}} s_{\mu} s_{\delta^{*}}=\sum_{\left\{\alpha \in \Lambda^{q-d(\lambda)}, \beta \in \Lambda^{q-d(\mu)}: \lambda \alpha=\mu \beta\right\}} s_{\sigma \alpha} s_{(\delta \beta)^{*}} .
$$

Since $H$ is hereditary, $r(\alpha)=s(\sigma)$ and $r(\beta)=s(\lambda)$ imply that $s(\alpha)$ and $s(\beta)$ are in $H$. Thus each nonzero summand in (5.2) belongs to $J$. Similarly, $s_{\mu} s_{\delta^{*}} s_{\sigma} s_{\lambda^{*}} \in J$. Thus $J$ is an ideal, and we have proved (5.1).

To see that $I_{H}$ is idempotent, we suppose that $s(\sigma)=s(\lambda) \in H$, and observe that the spanning element $s_{\sigma} s_{\lambda^{*}}=\left(s_{\sigma} p_{s(\sigma)}\right)\left(p_{s(\sigma)} s_{\lambda^{*}}\right)$ for $I_{H}$ belongs to $\left(I_{H}\right)^{2}$. Since (5.1) shows that $I_{H}$ is spanned by homogeneous elements, $I_{H}$ is graded.

To see that $I_{H}$ is basic and that $H=H_{I_{H}}$, it suffices to fix $r \neq 0$ in $R$, and prove that $v \notin H$ implies $r p_{v} \notin I_{H}$. Now consider the universal Kumjian-Pask $(\Lambda \backslash H)$-family $(q, t)$ in $\mathrm{KP}_{R}(\Lambda \backslash H)$, and extend it to a Kumjian-Pask $\Lambda$-family $(P, S)$ as in Lemma 5.3. The universal property of $\mathrm{KP}_{R}(\Lambda)$ (see Theorem 3.4) gives a homomorphism $\pi:=\pi_{P, S}: \mathrm{KP}_{R}(\Lambda) \rightarrow \mathrm{KP}_{R}(\Lambda \backslash H)$. Since $\pi\left(p_{w}\right)=0$ for $w \in H$, $\pi$ vanishes on $I_{H}$. On the other hand, applying Theorem 3.4 to $\Lambda \backslash H$ shows that $\pi\left(r p_{v}\right)=r q_{v} \neq 0$ for every $v \in \Lambda^{0} \backslash H$. Thus $r p_{v}$ cannot be in $I_{H} \subset \operatorname{ker} \pi$.

Proposition 5.5. Let $\Lambda$ be a row-finite $k$-graph without sources and $R$ a commutative ring with 1 . Let $I$ be a basic ideal of $\mathrm{KP}_{R}(\Lambda)$, and let $(q, t)$ and $(p, s)$ be the universal Kumjian-Pask families in $\mathrm{KP}_{R}\left(\Lambda \backslash H_{I}\right)$ and $\mathrm{KP}_{R}(\Lambda)$, respectively. If $I$ is graded or $\Lambda \backslash H_{I}$ is aperiodic, then there exists an isomorphism $\pi: \mathrm{KP}_{R}\left(\Lambda \backslash H_{I}\right) \rightarrow \mathrm{KP}_{R}(\Lambda) / I$ such that

$$
\pi\left(q_{v}\right)=p_{v}+I, \pi\left(t_{\lambda}\right)=s_{\lambda}+I \text { and } \pi\left(t_{\mu^{*}}\right)=s_{\mu^{*}}+I
$$

for $v \in \Lambda^{0} \backslash H_{I}$ and $\lambda, \mu \in \Lambda^{\neq 0} \cap s^{-1}\left(\Lambda^{0} \backslash H_{I}\right)$.

Proof. Observe that $\left\{p_{v}+I, s_{\lambda}+I, s_{\mu^{*}}+I\right\}$ is a Kumjian-Pask $\left(\Lambda \backslash H_{I}\right)$-family $(p+I, s+I)$, and the universal property of $\mathrm{KP}_{R}\left(\Lambda \backslash H_{I}\right)$ (Theorem 3.4) gives a homomorphism $\pi:=\pi_{p+I, s+I}$ satisfying (5.3). Since the other generators of $\operatorname{KP}_{R}(\Lambda)$ belong to $I$, the family $(p+I, s+I)$ generates $\mathrm{KP}_{R}(\Lambda) / I$, and $\pi$ is surjective.

Suppose that $\pi\left(r q_{v}\right)=0$ for some $r \in R \backslash\{0\}$ and $v \notin H_{I}$. Then $r p_{v}+I=$ $\pi\left(r q_{v}\right)=0$, so that $r p_{v} \in I$ and, since $I$ is basic, $p_{v} \in I$ as well. But this implies that $v \in H_{I}$, a contradiction. Thus $\pi\left(r q_{v}\right) \neq 0$ for all $r \in R \backslash\{0\}$ and $v \notin H_{I}$. If $\Lambda \backslash H_{I}$ is aperiodic, then the Cuntz-Krieger uniqueness theorem implies that $\pi$ is injective.

If $I$ is graded, then $\mathrm{KP}_{R}(\Lambda) / I$ is graded by $\left(\mathrm{KP}_{R}(\Lambda) / I\right)_{n}=q\left(\operatorname{KP}_{R}(\Lambda)_{n}\right)$, where $q: \mathrm{KP}_{R}(\Lambda) \rightarrow \mathrm{KP}_{R}(\Lambda) / I$ is the quotient map. If $\alpha, \beta \in\left(\Lambda \backslash H_{I}\right)$ with $d(\alpha)-d(\beta)=$ $n \in \mathbb{Z}^{k}$, then

$$
\pi\left(t_{\alpha} t_{\beta^{*}}\right)=s_{\alpha} s_{\beta^{*}}+I=q\left(s_{\alpha} s_{\beta^{*}}\right) \in q\left(\operatorname{KP}_{R}(\Lambda)_{n}\right)=\left(\operatorname{KP}_{R}(\Lambda) / I\right)_{n} .
$$

Thus $\pi$ is graded, and the graded-uniqueness theorem implies that $\pi$ is injective.

Proof of Theorem 5.1. To see that $H \mapsto I_{H}$ is surjective, let $I$ be a basic graded ideal. Then $H_{I}=\left\{v \in \Lambda^{0}: p_{v} \in I\right\}$ is saturated and hereditary by Lemma 5.2, Let $J:=I_{H_{I}}$. We will show that $I=J$. Since all the generators of $J$ lie in $I$, we have $J \subset I$. Consider the quotient map $Q: \mathrm{KP}_{R}(\Lambda) / J \rightarrow \mathrm{KP}_{R}(\Lambda) / I$. Since $H_{I_{H}}=H$ by 
Lemma 5.4. Proposition 5.5 gives us an isomorphism $\pi: \mathrm{KP}_{R}\left(\Lambda \backslash H_{I}\right) \rightarrow \mathrm{KP}_{R}(\Lambda) / J$. Now suppose $v$ belongs to $\Lambda^{0} \backslash H_{I}$ and $r \neq 0$. The composition $Q \circ \pi$ satisfies $Q \circ \pi\left(r q_{v}\right)=r p_{v}+I$, and since $I$ is basic,

$$
Q \circ \pi\left(r q_{v}\right)=0 \Longrightarrow r p_{v} \in I \Longrightarrow p_{v} \in I \Longrightarrow v \in H_{I},
$$

which contradicts the choice of $v$. So $Q \circ \pi\left(r q_{v}\right) \neq 0$, and it follows from the gradeduniqueness theorem (Theorem 4.1) that $Q \circ \pi$ is injective. Thus $Q$ is injective, and $I=J$. Thus $H \mapsto I_{H}$ is surjective.

Injectivity of $H \mapsto I_{H}$ follows because $H_{I_{H}}=H$ by Lemma 5.4. Finally, since $H \subset K$ if and only if $I_{H} \subset I_{K}$, the map $H \mapsto I_{H}$ preserves least upper bounds and greatest lower bounds, and hence is a lattice isomorphism.

The hypothesis that "every $\Lambda \backslash H$ is aperiodic" in the next theorem is the analogue for $k$-graphs of Condition (K) for directed graphs.

Theorem 5.6. Let $\Lambda$ be a row-finite $k$-graph without sources and let $R$ be a commutative ring with 1 . Then every basic ideal of $\mathrm{KP}_{R}(\Lambda)$ is graded if and only if $\Lambda \backslash H$ is aperiodic for every saturated hereditary subset $H$ of $\Lambda^{0}$.

Theorem 5.6 and Theorem 5.1 together have the following corollary.

Corollary 5.7. Let $\Lambda$ be a row-finite $k$-graph without sources and let $R$ be a commutative ring with 1 . Suppose that $\Lambda \backslash H$ is aperiodic for every saturated hereditary subset $H$ of $\Lambda^{0}$. Then $H \mapsto I_{H}$ is an isomorphism of the lattice of saturated hereditary subsets of $\Lambda^{0}$ onto the lattice of basic ideals in $\mathrm{KP}_{R}(\Lambda)$.

To prove Theorem 5.6 we need some more results. The next lemma is 32 , Lemma 3.3]; since the proof in [32] invokes results about a different formulation of periodicity, we give a direct proof.

Lemma 5.8. Suppose that $\Lambda$ is periodic. Then there exist $v \in \Lambda^{0}$ and $m \neq n \in \mathbb{N}^{k}$ such that, for all $\mu \in v \Lambda^{m}$ and $\alpha \in s(\mu) \Lambda^{(m \vee n)-m}$, there exists $\nu \in v \Lambda^{n}$ with $\mu \alpha y=\nu \alpha y$ for all $y \in s(\alpha) \Lambda^{\infty}$.

Proof. Since $\Lambda$ is periodic, there exist $v \in \Lambda^{0}$ and $m \neq n \in \mathbb{N}^{k}$ such that for all $\lambda \in v \Lambda$ with $d(\lambda) \geq m \vee n$ we have

$$
\lambda(m, m+d(\lambda)-(m \vee n))=\lambda(n, n+d(\lambda)-(m \vee n)) .
$$

For every $x \in v \Lambda^{\infty}$ and $l \in \mathbb{N}^{k}$, we can apply (5.4) to $\lambda=x(0,(m \vee n)+l)$, and deduce that $x(m, m+l)=x(n, n+l)$; in other words, for all $x \in v \Lambda^{\infty}$, we have $x(m, \infty)=x(n, \infty)$. Let $\mu \in v \Lambda^{m}$ and $\alpha \in s(\mu)^{(m \vee n)-m}$. Take $\nu=(\mu \alpha)(0, n)$, and let $y \in s(\alpha) \Lambda^{\infty}$. Then $x:=\mu \alpha y$ belongs to $v \Lambda^{\infty}$, and hence

$$
\begin{aligned}
\mu \alpha y & =(\mu \alpha y)(0, n)(\mu \alpha y)(n, \infty)=(\mu \alpha)(0, n)(\mu \alpha y)(n, \infty) \\
& =\nu(\mu \alpha y)(n, \infty)=\nu(\mu \alpha y)(m, \infty)=\nu \alpha y .
\end{aligned}
$$

The following lemma is used in the proofs of Proposition 5.11 and Theorem 5.6.

Lemma 5.9. Let $\pi_{Q, T}: \operatorname{KP}_{R}(\Lambda) \rightarrow \operatorname{End}\left(\mathbb{F}_{R}\left(\Lambda^{\infty}\right)\right)$ be the infinite-path representation from the end of $\S 3$. If $\Lambda$ is periodic, then there exist $\mu, \nu, \alpha \in \Lambda$ such that

$$
0 \neq s_{\mu \alpha} s_{(\mu \alpha) *}-s_{\nu \alpha} s_{(\mu \alpha)} \in \operatorname{ker} \pi_{Q, T} .
$$


Proof. Take $v \in \Lambda^{0}, m \neq n \in \mathbb{N}^{k}$ as given by Lemma [5.8, and choose $\mu \in v \Lambda^{m}$ and $\alpha \in s(\mu) \Lambda^{(m \vee n)-m}$. Then there exists $\nu \in v \Lambda^{n}$ such that $\mu \alpha y=\nu \alpha y$ for all $y \in \Lambda^{\infty}$. Suppose, by way of contradiction, that $a:=s_{\mu \alpha} s_{(\mu \alpha)^{*}}-s_{\nu \alpha} s_{(\mu \alpha)^{*}}=0$. Then $s_{\mu \alpha} s_{(\mu \alpha)^{*}}=s_{\nu \alpha} s_{(\mu \alpha)^{*}}$. But $d\left(s_{\mu \alpha} s_{(\mu \alpha)^{*}}\right)=d(\mu \alpha)-d(\mu \alpha)=0$, whereas

$$
d\left(s_{\nu \alpha} s_{(\mu \alpha) *}\right)=d(\nu \alpha)-d(\mu \alpha)=d(\nu)+d(\alpha)-d(\mu)-d(\alpha)=n-m \neq 0 .
$$

Thus $s_{\mu \alpha} s_{(\mu \alpha)^{*}}=s_{\nu \alpha} s_{(\mu \alpha)^{*}}=0$. But now $0=s_{(\mu \alpha)^{*}}\left(s_{\mu \alpha} s_{(\mu \alpha) *}\right) s_{\mu \alpha}=p_{s(\mu \alpha)}^{2}=$ $p_{s(\alpha)}$ contradicts Theorem 3.4. Hence $a \neq 0$.

To see that $a \in \operatorname{ker} \pi_{Q, T}$ we fix $x \in \Lambda^{\infty}$ and show that $\pi_{Q, T}(a)(x)=0$. Recall that $\pi_{Q, T}\left(s_{\lambda}\right)=T_{\lambda}$ and $\pi_{Q, T}\left(s_{\lambda^{*}}\right)=T_{\lambda^{*}}$, where

$$
T_{\lambda}(x)=\left\{\begin{array}{ll}
\lambda x & \text { if } x(0)=s(\lambda) \\
0 & \text { otherwise }
\end{array} \text { and } T_{\lambda^{*}}(x)= \begin{cases}x(d(\lambda), \infty) & \text { if } x(0, d(\lambda))=\lambda \\
0 & \text { otherwise }\end{cases}\right.
$$

If $x(0, d(\mu \alpha)) \neq \mu \alpha$, then $T_{(\mu \alpha)^{*}}(x)=0$ and hence $\pi_{Q, T}(a)(x)=T_{\mu \alpha} T_{(\mu \alpha)^{*}}(x)-$ $T_{\nu \alpha} T_{(\mu \alpha) *}(x)=0$. On the other hand, if $x(0, d(\mu \alpha))=\mu \alpha$, then $\pi_{Q, T}(a)(x)=$ $\left(T_{\mu \alpha}-T_{\nu \alpha}\right)(x(d(\mu \alpha), \infty))$ has the form $\mu \alpha y-\nu \alpha y$ for $y=x(d(\mu \alpha), \infty)$, and hence $\pi_{Q, T}(a)(x)=0$. Thus $a \in \operatorname{ker} \pi_{Q, T}$.

Corollary 5.10. Suppose that $\Lambda$ is a row-finite $k$-graph without sources. Then the infinite-path representation $\pi_{Q, T}$ from the end of $\S 3$ is injective if and only if $\Lambda$ is aperiodic.

Proof. Lemma 5.9 shows that $\operatorname{ker} \pi_{Q, T}$ is nonzero when $\Lambda$ is periodic, and the converse is Corollary 4.10 .

Proposition 5.11. Let $\Lambda$ be a row-finite $k$-graph without sources, and let $R$ be a commutative ring with 1 . Then $\Lambda$ is aperiodic if and only if every nonzero basic ideal of $\mathrm{KP}_{R}(\Lambda)$ contains a vertex idempotent $p_{v}$.

Proof. If $\Lambda$ is periodic, then we know from Lemma 5.9 that the kernel of the infinitepath representation is nonzero; it is basic because it contains no $r p_{v}$ where $r \neq 0$ (by construction). So suppose that $\Lambda$ is aperiodic, and $I$ is a basic ideal in $\operatorname{KP}_{R}(\Lambda)$ such that $p_{v} \notin I$ for all $v \in \Lambda^{0}$; we want to show that $I=\{0\}$.

If either $s_{\lambda} \in I$ or $s_{\lambda^{*}} \in I$, then $p_{s(\lambda)}=s_{\lambda^{*}} s_{\lambda} \in I$, contradicting the assumption. Thus $p_{v}+I, s_{\lambda}+I, s_{\mu^{*}}+I$ are nonzero for all $v \in \Lambda^{0}$ and $\lambda, \mu \in \Lambda^{\neq 0}$, and they form a Kumjian-Pask $\Lambda$-family in $\mathrm{KP}_{R}(\Lambda) / I$ which induces a surjective homomorphism $\pi_{p+I, s+I}: \mathrm{KP}_{R}(\Lambda) \rightarrow \mathrm{KP}_{R}(\Lambda) / I$ such that $\pi_{p+I, s+I}\left(p_{v}\right)=p_{v}+I$.

Suppose that $\pi_{p+I, s+I}\left(r p_{v}\right)=0$ for some $r \in R \backslash\{0\}$. Then $0=\pi_{p+I, s+I}\left(r p_{v}\right)=$ $r\left(p_{v}+I\right)$ implies that $r p_{v} \in I$, and, since $I$ is basic, this implies $p_{v} \in I$, a contradiction. Thus $\pi_{p+I, s+I}\left(r p_{v}\right) \neq 0$ for all $r \in R \backslash\{0\}$. Since $\Lambda$ is aperiodic, the Cuntz-Krieger uniqueness theorem implies that $\pi_{p+I, s+I}$ is an isomorphism. But $\pi_{p+I, s+I}$ is the quotient map, and hence $I=\{0\}$, as required.

Proof of Theorem 5.6. Suppose that $\Lambda^{0}$ contains a saturated hereditary subset $H$ such that $\Lambda \backslash H$ is periodic. Let $(q, t)$ be the universal Kumjian-Pask $\Lambda \backslash H$ family in $\operatorname{KP}_{R}(\Lambda \backslash H)$. Then Lemma 5.9 implies that the kernel of the infinite-path representation is a nonzero ideal in $\mathrm{KP}_{R}(\Lambda \backslash H)$ which contains no $r q_{v}$, and pulling this ideal over under the isomorphism of Proposition 5.5 gives an ideal $K$ in $\operatorname{KP}_{R}(\Lambda) / I_{H}$ which contains no $r\left(p_{v}+I_{H}\right)$ for $r \neq 0$ and $v \notin H$. But then the inverse image of 
$K$ in $\mathrm{KP}_{R}(\Lambda)$ is an ideal $J$ which strictly contains $I_{H}$ and satisfies

$$
\begin{aligned}
r p_{v} \in J \text { for some } r \neq 0 & \Longrightarrow r\left(p_{v}+I_{H}\right) \in K \text { for some } r \neq 0 \\
& \Longrightarrow p_{v} \in J \\
& \Longrightarrow v \in H .
\end{aligned}
$$

These implications show, first, that $J$ is basic, and, second, that $H_{J}=H$. But then $J \neq I_{H_{J}}=I_{H}$, and $J$ cannot be graded by Theorem 5.1 ,

Conversely, suppose that every $\Lambda \backslash H$ is aperiodic, and that $J$ is a nonzero basic ideal of $\mathrm{KP}_{R}(\Lambda)$. We trivially have $I_{H_{J}} \subset J$, and we claim that in fact $I_{H_{J}}=J$. Suppose not. Then $J / I_{H_{J}}$ is a nonzero ideal in $\mathrm{KP}_{R}(\Lambda) / I_{H_{J}}$, and its inverse image $L$ under the isomorphism of Proposition [5.5] is a nonzero ideal in $\operatorname{KP}_{R}\left(\Lambda \backslash H_{J}\right)$. This ideal $L$ is basic: if $r \neq 0$ and $q_{v}$ is a vertex idempotent in $\operatorname{KP}_{R}\left(\Lambda \backslash H_{J}\right)$, then

$$
r q_{v} \in L \Longrightarrow r p_{v}+I_{H_{J}} \in J / I_{H_{J}} \Longrightarrow r p_{v} \in J \Longrightarrow p_{v} \in J \Longrightarrow q_{v} \in L .
$$

Since $\Lambda \backslash H_{J}$ is aperiodic, Proposition 5.11 implies that $L$ contains some $q_{v}$ for $v \in \Lambda^{0} \backslash H_{J}$. But then $J$ contains $p_{v}$, and $v \in H_{J}$, which is a contradiction. Thus $J=I_{H_{J}}$, and Lemma 5.4 implies that $J$ is graded.

As in [38, we say that $\mathrm{KP}_{R}(\Lambda)$ is basically simple if its only basic ideals are $\{0\}$ and $\mathrm{KP}_{R}(\Lambda)$. If $R$ is a field, then every ideal is basic, and hence basic simplicity is the same as simplicity.

Our next goal is to obtain necessary and sufficient conditions for the basic simplicity of $\mathrm{KP}_{R}(\Lambda)$. We do this independently of Theorem 5.1 by following the approach of 32. A $k$-graph $\Lambda$ is cofinal if for every $x \in \Lambda^{\infty}$ and every $v \in \Lambda^{0}$, there exists $n \in \mathbb{N}^{k}$ such that $v \Lambda x(n) \neq \emptyset$. This cofinality condition is based on the one used for directed graphs in [23, §3].

Lemma 5.12. If $\Lambda$ is cofinal, then the only saturated hereditary subsets of $\Lambda^{0}$ are $\emptyset$ and $\Lambda^{0}$.

Proof. Suppose there exists a nontrivial saturated hereditary subset $H$ of $\Lambda^{0}$. Choose $v \in \Lambda^{0} \backslash H$ and $w \in H$. Choose a sequence $\{n(i)\}$ in $\mathbb{N}^{k}$ such that $n(i) \leq n(i+1)$ and $n(i) \rightarrow \infty$ in the sense that $n(i)_{j} \rightarrow \infty$ as $i \rightarrow \infty$ for $1 \leq j \leq k$. Since $v \notin H$ and $H$ is saturated, there exists $\lambda_{1} \in v \Lambda^{n(1)}$ such that $s\left(\lambda_{1}\right) \notin H$. By induction, for $i \geq 1$ there exists $\lambda_{i+1} \in s\left(\lambda_{i}\right) \Lambda^{n(i+1)-n(i)}$ such that $s\left(\lambda_{i+1}\right) \notin H$. Now set $\mu_{1}=\lambda_{1}$ and $\mu_{i+1}=\mu_{i} \lambda_{i+1}$ for $i \geq 1$. Then $\mu_{i+1}(0, n(i))=\mu_{i}$, and by Lemma 2.4 there exists $y \in \Lambda^{\infty}$ such that $y(0, n(i))=\mu_{i}=\lambda_{1} \ldots \lambda_{i}$.

Since $\Lambda$ is cofinal, there exists $m \in \mathbb{N}^{k}$ such that $w \Lambda y(m) \neq \emptyset$. Since $w \in H$ and $H$ is hereditary, we have $y(m) \in H$. Choose $i_{0} \in \mathbb{N}$ such that $n\left(i_{0}\right) \geq m$. Then $y\left(n\left(i_{0}\right)\right)=s\left(\lambda_{i_{0}}\right)$ belongs to $H$ because $H$ is hereditary. But $s\left(\lambda_{i_{0}}\right) \notin H$ by construction, and we have a contradiction. So the only saturated hereditary subsets are the trivial ones.

Proposition 5.13. Let $\Lambda$ be a row-finite k-graph without sources, and let $R$ be a commutative ring with 1 . Then $\Lambda$ is cofinal if and only if the only basic ideal containing a vertex idempotent $p_{v}$ is $\mathrm{KP}_{R}(\Lambda)$.

Proof. Suppose that $\Lambda$ is cofinal, and $I$ is a basic ideal containing some $p_{w}$. Then $H_{I}=\left\{v \in \Lambda^{0}: p_{v} \in I\right\}$ is nonempty, and is saturated and hereditary by Lemma 
5.2. Since $\Lambda$ is cofinal, $H_{I}=\Lambda^{0}$ by Lemma 5.12. Thus $p_{v} \in I$ for all $v \in \Lambda^{0}$, and we have

$$
\mathrm{KP}_{R}(\Lambda)=\operatorname{span}\left\{s_{\alpha} p_{s(\alpha)} s_{\beta^{*}}: \alpha, \beta \in \Lambda^{\neq 0}, s(\alpha)=s(\beta)\right\} \subset I .
$$

Now suppose that $\Lambda$ is not cofinal. Then there exist $v \in \Lambda^{0}$ and an infinite path $x \in \Lambda^{\infty}$ such that $v \Lambda x(n)=\emptyset$ for every $n \in \mathbb{N}^{k}$. By [32, Proposition 3.4, proof of (ii) $\Rightarrow$ (i)] the set $H_{x}:=\left\{w \in \Lambda^{0}: w \Lambda x(n)=\emptyset\right.$ for all $\left.n \in \mathbb{N}^{k}\right\}$ is a saturated hereditary subset of $\Lambda^{0}$. Note that $H_{x}$ is nontrivial since $v \in H_{x}$ and $x(0) \notin H_{x}$. Now $I_{H_{x}}$ is a basic ideal of $\mathrm{KP}_{R}(\Lambda)$ by Lemma 5.4 and $p_{v} \in I_{H_{x}}$. But $H_{I_{H_{x}}}=H_{x}$ by Lemma [5.4, and hence $p_{x(0)} \notin I_{H_{x}}$ because $x(0) \notin H_{x}$. Thus $I_{H_{x}} \neq \operatorname{KP}_{R}(\Lambda)$, and we have a nontrivial ideal containing a vertex idempotent.

Theorem 5.14. Let $\Lambda$ be a row-finite $k$-graph without sources, and let $R$ be a commutative ring with 1 . Then $\mathrm{KP}_{R}(\Lambda)$ is basically simple if and only if the graph $\Lambda$ is cofinal and aperiodic.

Proof. If $\mathrm{KP}_{R}(\Lambda)$ is basically simple, then the only nonzero basic ideal is $\operatorname{KP}_{R}(\Lambda)$. So Proposition 5.11 implies that $\Lambda$ is aperiodic, and Proposition 5.13 implies that $\Lambda$ is cofinal.

Conversely, assume that $\Lambda$ is cofinal and aperiodic and $I$ is a nonzero basic ideal in $\operatorname{KP}_{R}(\Lambda)$. By Proposition 5.11 there exists $v \in \Lambda^{0}$ with $p_{v} \in I$. But then $I=\mathrm{KP}_{R}(\Lambda)$ by Proposition 5.13. Thus $\mathrm{KP}_{R}(\Lambda)$ is basically simple.

Remark 5.15. The parametrization of ideals in Cuntz-Krieger algebras by the saturated hereditary subsets comes from [11], and was extended to various classes of graph $C^{*}$-algebras in [23, 9, 8, 18, The ideals in the $C^{*}$-algebras of higher-rank graphs were first analyzed in [30]. The graded ideals in the Leavitt path algebras were described in 5, 37] and 38. The simplicity theorem for $C^{*}$-algebras goes back to Cuntz and Krieger [12, and for Leavitt path algebras to Abrams and Aranda Pino 1]. Our proof of basic simplicity was inspired by the work of Robertson and Sims [32.

\section{Simplicity}

Let $\Lambda$ be a row-finite $k$-graph without sources, and write $(p, s)$ for the universal Kumjian-Pask family in $\mathrm{KP}_{R}(\Lambda)$. So far the $\operatorname{ring} R$ has played little role in our study of $\mathrm{KP}_{R}(\Lambda)$; in fact, the notion of a basic ideal in the previous section was engineered by Tomforde to avoid dealing with ideals in $R$. The main result of this section is:

Theorem 6.1. Suppose that $\Lambda$ is a row-finite $k$-graph without sources, and that $R$ is a commutative ring with 1 . Then $\mathrm{KP}_{R}(\Lambda)$ is simple if and only if $R$ is a field and $\Lambda$ is aperiodic and cofinal.

This theorem was motivated by the following observations. If $R$ is an algebra over a commutative ring $S$, then [38, Theorem 8.1] implies that $L_{R}(E)$ is isomorphic to $R \otimes_{S} L_{S}(E)$ as an $R$-algebra. Moreover, if $A$ is an $s$-unital algebra over a field $K$, and $E$ is a cofinal graph in which every cycle has an entry, then 7 , Corollary 7.8] implies that every ideal of $A \otimes_{K} L_{K}(E)$ has the form $I \otimes_{K} L_{K}(E)$ for some ideal $I$ of $A$. 
We write $\mathcal{L}(A)$ for the lattice of ideals of a ring $A$. Then we can define restriction and induction maps

$$
\text { Res : } \mathcal{L}\left(\mathrm{KP}_{R}(\Lambda)\right) \rightarrow \mathcal{L}(R) \text { and } \quad \text { Ind }: \mathcal{L}(R) \rightarrow \mathcal{L}\left(\mathrm{KP}_{R}(\Lambda)\right)
$$

as follows:

$$
\begin{aligned}
\operatorname{Res} I & :=\left\{r \in R: r p_{v} \in I \text { for all } v \in \Lambda^{0}\right\}, \\
\text { Ind } M & :=\operatorname{span}_{R}\left\{r s_{\alpha} s_{\beta}^{*}: r \in M, \alpha, \beta \in \Lambda\right\} .
\end{aligned}
$$

One can easily check that Res $I$ and Ind $M$ are ideals in $R$ and $\operatorname{KP}_{R}(\Lambda)$, respectively.

We will need the following lemma in Proposition 6.3 and in Proposition 6.4.

Lemma 6.2. Let $M$ be an ideal of $R, r \in R$, and $v \in \Lambda^{0}$. If $r p_{v} \in$ Ind $M$, then $r \in M$.

Proof. If $r p_{v}=0$, then $r=0$ and is in $M$. So suppose $r p_{v} \neq 0$. We have $r p_{v}=\sum_{(\alpha, \beta) \in F} r_{\alpha, \beta} s_{\alpha} s_{\beta^{*}}$ for some $r_{\alpha, \beta} \in M \backslash\{0\}$; by Lemma 4.2 we may assume this is in normal form, and a glance at the proof of Lemma 4.2 shows that the $r_{\alpha, \beta}$ are then still in $M \backslash\{0\}$. By Lemma 4.3 there exists $\gamma \in \Lambda$ and a finite set $G \subset \Lambda$ such that $0 \neq r p_{v} s_{\gamma}=\sum_{\alpha \in G} r_{\alpha, \gamma} s_{\alpha}$. Since $\operatorname{KP}_{R}(\Lambda)$ is $\mathbb{Z}^{k}$-graded we have

$$
0 \neq\left(r p_{v}\right) s_{\gamma}=\sum_{\{\alpha \in G: d(\alpha)=d(\gamma)\}} r_{\alpha, \gamma} s_{\alpha} .
$$

We must have $v=r(\gamma)$, and applying (KP3) gives

$$
\begin{aligned}
r p_{s(\gamma)}=r s_{\gamma^{*}} s_{\gamma} & =s_{\gamma^{*}}\left(r p_{v}\right) s_{\gamma}=\sum_{\{\alpha \in G: d(\alpha)=d(\gamma)\}} r_{\alpha, \gamma} s_{\gamma^{*}} s_{\alpha} \\
& = \begin{cases}r_{\gamma, \gamma} p_{s(\gamma)} & \text { if } \gamma \in G, \\
0 & \text { otherwise. }\end{cases}
\end{aligned}
$$

But now either $\left(r-r_{\gamma, \gamma}\right) p_{s(\gamma)}=0$ or $r p_{s(\gamma)}=0$, and hence either $r=r_{\gamma, \gamma}$ or $r=0$ by Theorem 3.4. In either case, $r \in M$.

Proposition 6.3. Suppose that $\Lambda$ is a row-finite $k$-graph without sources, that $R$ is a commutative ring with 1 and that $M$ is a proper ideal of $R$. Then $\operatorname{KP}_{R}(\Lambda) / \operatorname{Ind} M$ is an $R / M$-algebra with $(r+M)(x+\operatorname{Ind} M)=r x+\operatorname{Ind} M$, and there is an isomorphism $\pi$ of $\mathrm{KP}_{R / M}(\Lambda)$ onto $\mathrm{KP}_{R}(\Lambda) /$ Ind $M$ which takes the universal KumjianPask family $(q, t)$ in $\mathrm{KP}_{R / M}(\Lambda)$ to $(p+\operatorname{Ind} M, s+$ Ind $M)$.

Proof. To see that the action of $R / M$ is well-defined, note that if $r+M=s+M$ and $x+\operatorname{Ind} M=y+\operatorname{Ind} M$, then

$$
r x-s y=r(x-y)+(r-s) y \in R \cdot \text { Ind } M+M \cdot \operatorname{KP}_{R}(\Lambda) \subset \text { Ind } M,
$$

as required.

The pair $(p+\operatorname{Ind} M, s+\operatorname{Ind} M)$ is a Kumjian-Pask family in $\operatorname{KP}_{R}(\Lambda) / \operatorname{Ind} M$, and thus the universal property of $\mathrm{KP}_{R / M}(\Lambda)$ (Theorem 3.4) gives a homomorphism $\pi$ taking $(q, t)$ to $(p+\operatorname{Ind} M, s+\operatorname{Ind} M) ; \pi$ is surjective because $(p, s)$ generates $\mathrm{KP}_{R}(\Lambda)$. The ideal Ind $M$ is spanned by homogeneous elements, and hence is graded; then $\operatorname{KP}_{R}(\Lambda) /$ Ind $M$ is graded by the images $q\left(\operatorname{KP}_{R}(\Lambda)_{n}\right)$ under the quotient map $q$. The homomorphism $\pi$ is then a graded homomorphism. Since $M$ is proper, Lemma 6.2 implies that no vertex projection $p_{v}$ belongs to Ind $M$, and hence each vertex projection $p_{v}+$ Ind $M$ in the quotient is nonzero. Thus the graded-uniqueness theorem implies that $\pi$ is injective. 
Proposition 6.4. Let $\Lambda$ be a row-finite k-graph without sources, and let $R$ be a commutative ring with 1 .

(a) We have Res $\circ$ Ind = id. In particular, Ind is injective.

(b) Suppose that $\Lambda$ is aperiodic and cofinal. Then Ind $\circ$ Res $=\mathrm{id}$, and Ind : $\mathcal{L}(R) \rightarrow \mathcal{L}\left(\mathrm{KP}_{R}(\Lambda)\right)$ is a lattice isomorphism with inverse Res.

Proof. (国) Let $M$ be an ideal of $R$. We will show that $\operatorname{Res} \circ \operatorname{Ind}(M)=M$, and the injectivity of Ind then follows. If $m \in M$, then $m p_{v} \in \operatorname{Ind} M$ for all $v \in \Lambda^{0}$, and hence $m \in \operatorname{Res} \circ$ Ind $M$. Thus $M \subset \operatorname{Res} \circ \operatorname{Ind} M$. For the reverse inclusion, let $t \in \operatorname{Res} \circ$ Ind $M$. Then $t p_{v} \in$ Ind $M$ for $v \in \Lambda^{0}$ and hence $t \in M$ by Lemma 6.2.

(b) Let $I$ be a nonzero ideal of $\operatorname{KP}_{R}(\Lambda)$. We will show that Ind $\circ \operatorname{Res} I=I$, and the surjectivity of Ind then follows. Let $0 \neq x \in I$. We write $x$ in normal form $\sum_{(\alpha, \beta) \in F} r_{\alpha, \beta} s_{\alpha} s_{\beta^{*}}$ (see Lemma 4.2). Since $\Lambda$ is aperiodic, by Proposition 4.9 there exist $\sigma, \tau \in \Lambda$ and $(\delta, \gamma) \in F$ such that $s_{\sigma^{*}} x s_{\tau}=r_{\delta, \gamma} p_{w}$ for some $w \in \Lambda^{0}$. Then $r_{\delta, \gamma} p_{w} \in I$, and thus $w$ is in the saturated hereditary subset $H_{I, r_{\delta, \gamma}}$ of Lemma [5.2. Since $\Lambda$ is cofinal by hypothesis, Lemma 5.12 implies that $H_{I, r_{\delta, \gamma}}=\Lambda^{0}$, so that $r_{\delta, \gamma} p_{v} \in I$ for all $v \in \Lambda^{0}$. In particular, $r_{\delta, \gamma} p_{r(\delta)} \in I$, and hence

$$
y:=x-r_{\delta, \gamma} p_{r(\delta)} s_{\delta} s_{\gamma^{*}}=\sum_{(\alpha, \beta) \in F \backslash\{(\delta, \gamma)\}} r_{\alpha, \beta} s_{\alpha} s_{\beta^{*}}
$$

belongs to $I$ and is in normal form. Repeating the above process $|F|-1$ times gives $r_{\alpha, \beta} p_{v} \in I$ for all $v \in \Lambda^{0}$ and $(\alpha, \beta) \in F$. Thus $r_{\alpha, \beta} \in \operatorname{Res} I$ for $(\alpha, \beta) \in F$, and hence $x \in \operatorname{Ind} \circ \operatorname{Res} I$. Thus $I \subset$ Ind $\circ \operatorname{Res} I$.

For the reverse inclusion, let $y \in \operatorname{Ind} \circ \operatorname{Res} I$. Then $y=\sum r_{\alpha, \beta} s_{\alpha} s_{\beta^{*}}$, where each $r_{\alpha, \beta} \in \operatorname{Res} I$, that is, $r_{\alpha, \beta} p_{v} \in I$ for all $v \in \Lambda^{0}$. But now $y=\sum s_{\alpha}\left(r_{\alpha, \beta} p_{s(\alpha)}\right) s_{\beta^{*}} \in I$. Thus Ind $\circ \operatorname{Res} I=I$, and Ind is surjective. Since Ind is injective by (a), and since $M_{1} \subset M_{2}$ if and only Ind $M_{1} \subset$ Ind $M_{2}$, it follows that Ind is a lattice isomorphism.

Proof of Theorem 6.1. First suppose that $\mathrm{KP}_{R}(\Lambda)$ is simple. Then $\mathrm{KP}_{R}(\Lambda)$ is basically simple, and hence $\Lambda$ is aperiodic and cofinal by Theorem 5.14. Let $M$ be a nonzero ideal of $R$. Then Ind $M$ is a nonzero ideal of $\operatorname{KP}_{R}(\Lambda)$, and hence Ind $M=\mathrm{KP}_{R}(\Lambda)$. By Proposition 6.4(国), $M=\operatorname{Res} \circ \operatorname{Ind} M=\operatorname{Res} \mathrm{KP}_{R}(\Lambda)=R$. Thus $R$ is a field.

Conversely, assume that $\Lambda$ is aperiodic and cofinal, and that $R$ is a field. Let $I$ be a nonzero ideal of $\operatorname{KP}_{R}(\Lambda)$. Since $\Lambda$ is aperiodic and cofinal, by Proposition 6.4(b) we have $I=\operatorname{Ind} \circ \operatorname{Res} I$. Thus $\operatorname{Res} I$ is a nonzero ideal of $R$, and hence $\operatorname{Res} I=R$ since $R$ is simple. But now $I=\operatorname{Ind} R=\operatorname{KP}_{R}(\Lambda)$. Thus $\operatorname{KP}_{R}(\Lambda)$ is simple.

The next result is a converse for Proposition 6.4(b).

Proposition 6.5. Let $\Lambda$ be a row-finite k-graph without sources and let $R$ be a commutative ring with 1 . Then $\Lambda$ is aperiodic and cofinal if and only if Ind $\circ$ Res $=$ id.

Proof. Proposition 6.4(b) is the "only if" half. Suppose that Ind $\circ$ Res $=$ id. It suffices by Theorem 5.14 to prove that $\operatorname{KP}_{R}(\Lambda)$ is basically simple. So let $I$ be a nonzero basic ideal of $\operatorname{KP}_{R}(\Lambda)$. Then Ind $\circ \operatorname{Res} I=I$ implies that $\operatorname{Res} I$ is a nonzero ideal. Let $0 \neq r \in \operatorname{Res} I$. Then $r p_{v} \in I$ for all $v \in \Lambda^{0}$, and since $I$ is basic, $p_{v} \in I$ for all $v \in \Lambda^{0}$, and $I=\mathrm{KP}_{R}(\Lambda)$. Thus $\mathrm{KP}_{R}(\Lambda)$ is basically simple, as required. 


\section{EXAMPLES AND APPLICATIONS}

We begin with the easiest nontrivial example.

Example 7.1. Let $R$ be a commutative ring with 1. View $\Lambda=\mathbb{N}^{2}$ as a category with a single object $v$, and let $d: \mathbb{N}^{2} \rightarrow \mathbb{N}^{2}$ be the identity map. Then $\Lambda$ is the unique 2-graph whose skeleton consists of one blue and one red loop at a single vertex. For each $n \in \mathbb{N}^{2}$ there is a unique path $n$ of degree $n$, and a Kumjian-Pask family $(P, S)$ in an $R$-algebra must satisfy

$$
\begin{gathered}
P_{v}^{2}=P_{v}=S_{n^{*}} S_{n}=S_{n} S_{n^{*}}, \\
S_{m} S_{n}=S_{m+n}, S_{n^{*}} S_{m^{*}}=S_{(m+n)^{*}}, \\
P_{v} S_{n}=S_{n}=S_{n} P_{v}, P_{v} S_{n^{*}}=S_{n^{*}}=S_{n^{*}} P_{v} .
\end{gathered}
$$

For $q \geq m \vee n$ in $\mathbb{N}^{2}$, the sum in Lemma 3.3 has exactly one term, and we have $S_{m^{*}} S_{n}=S_{q-m} S_{(q-n)^{*}}$; taking $q=m+n$ gives $S_{m^{*}} S_{n}=S_{n} S_{m^{*}}$. In particular, $\mathrm{KP}_{R}(\Lambda)$ is commutative. We will use the graded-uniqueness theorem to show that $\mathrm{KP}_{R}(\Lambda)$ is isomorphic to the ring $R\left[x, x^{-1}, y, y^{-1}\right]$ of Laurent polynomials over $R$ in two commuting indeterminates $x$ and $y$.

Set $Q_{v}=1, T_{(i, j)}=x^{i} y^{j}$ and $T_{(i, j)^{*}}=x^{-i} y^{-j}$. Then $(Q, T)$ is a KumjianPask $\Lambda$-family in $R\left[x, x^{-1}, y, y^{-1}\right]$, and the universal property of $\operatorname{KP}_{R}(\Lambda)$ gives a homomorphism $\phi: \mathrm{KP}_{R}(\Lambda) \rightarrow R\left[x, x^{-1}, y, y^{-1}\right]$ such that $\phi \circ p=Q$ and $\phi \circ s=T$. The groups $A_{(i, j)}:=\operatorname{span}\left\{x^{i} y^{j}\right\}$ for $(i, j) \in \mathbb{Z}^{2}$ grade $R\left[x, x^{-1}, y, y^{-1}\right]$ over $\mathbb{Z}^{2}$, and $\phi$ maps $\operatorname{KP}_{R}(\Lambda)_{(i, j)}=\operatorname{span}\left\{s_{n} s_{m^{*}}: n-m=(i, j)\right\}$ into $A_{(i, j)}$, so $\phi$ is graded. Finally, $\phi\left(r p_{v}\right)=r \phi\left(p_{v}\right)=r 1=r \neq 0$ for all $r \in R \backslash\{0\}$, and so Theorem 4.1 implies that $\phi$ is injective. Since the image of $\phi$ contains a generating set for $R\left[x, x^{-1}, y, y^{-1}\right], \phi$ is an isomorphism.

Remark 7.2. Let $K$ be a field. We claim that $K\left[x, x^{-1}, y, y^{-1}\right]$ cannot be realized as a Leavitt path algebra $L_{K}(E)$ for any directed graph $E$. Thus Example 7.1 shows that the class of Kumjian-Pask algebras over $K$ is larger than the class of Leavitt path algebras over $K$. To see the claim, recall from [6, Proposition 2.7] that every commutative Leavitt path algebra has the form $\left(\bigoplus_{i \in I} K\right) \oplus\left(\bigoplus_{j \in J} K\left[x, x^{-1}\right]\right)$. Since $K\left[x, x^{-1}, y, y^{-1}\right]$ has no zero divisors, if $K\left[x, x^{-1}, y, y^{-1}\right]$ had this form, then it would be isomorphic to either $K$ or $K\left[x, x^{-1}\right]$ as rings. But both $K$ and $K\left[x, x^{-1}\right]$ are principal ideal domains, whereas $K\left[x, x^{-1}, y, y^{-1}\right]$ is not. So $K\left[x, x^{-1}, y, y^{-1}\right]$ is not the Leavitt path algebra of any directed graph.

7.1. The Kumjian-Pask algebra and the $C^{*}$-algebra. We have said that the graded-uniqueness theorem is an analogue for Kumjian-Pask algebras of the gaugeinvariant uniqueness theorem for graph $C^{*}$-algebras. Indeed, an original motivation for graded-uniqueness theorems was to prove that the Leavitt path algebra $L_{\mathbb{C}}(E)$ embeds in the graph $C^{*}$-algebra $C^{*}(E)$, and the proof of this inevitably uses the gauge action alongside the grading of $\mathrm{KP}_{\mathbb{C}}(\Lambda)$. Since the existing treatments $(29$, Corollary 1.3.3] and [37, Theorem 7.3]) are on the terse side, it seems worthwhile to give a careful treatment of the analogous result for Kumjian-Pask algebras.

When the coefficient ring $R$ is the field $\mathbb{C}$, the Kumjian-Pask algebra $\operatorname{KP}_{\mathbb{C}}(\Lambda)$ has a conjugate linear involution characterized in terms of the generating KumjianPask family by $\left(c s_{\lambda} s_{\mu^{*}}\right)^{*}=\bar{c} s_{\mu} s_{\lambda^{*}}$ for $c \in \mathbb{C}$. (To see this, we define $a \mapsto a^{*}$ on $\mathbb{F}_{\mathbb{C}}(w(X))$ by the analogous formula on infinite sums, check that this map is an involution on $\mathbb{F}_{\mathbb{C}}(w(X))$, and then observe that the ideal $I$ defined in the proof of 
Theorem 3.4 is ${ }^{*}$-closed, so the involution passes to the quotient $\operatorname{KP}_{\mathbb{C}}(\Lambda)$.) Thus $\mathrm{KP}_{\mathbb{C}}(\Lambda)$ is a $*$-algebra.

The $C^{*}$-algebra $C^{*}(\Lambda)$ is generated by a universal Cuntz-Krieger family $(q, t)$ of the sort described at the start of $\$ 3$. It is not completely obvious that such a $C^{*}$-algebra exists (though you'd never guess this to look at the literature!). But if we take the *-algebra $A$ generated by symbols $\left\{q_{v}, t_{e}\right\}$ subject to the relations, then because the elements $q_{v}$ and $t_{e}$ are all partial isometries, every generator has norm at most 1 in every representation of $A$ as bounded operators on Hilbert space; we can then define a semi-norm on $A$ by

$$
\|a\|=\sup \{\|\pi(a)\|: \pi: A \rightarrow B(H) \text { is a } * \text {-representation of } A\},
$$

mod out by the ideal of elements of norm 0 to get a normed algebra, and complete in the norm to get a $C^{*}$-algebra [10, $\left.\S 1\right]$. To see that this $C^{*}$-algebra is nonzero, Kumjian and Pask built a Cuntz-Krieger family on $\ell^{2}\left(\Lambda^{\infty}\right)$ in which every generator is nonzero, so in particular each $q_{v}$ is nonzero in $C^{*}(\Lambda)$ [21, Proposition 2.11].

As we saw at the start of $\oint 3$, the universal Cuntz-Krieger family $(q, t)$ in $C^{*}(\Lambda)$ is a Kumjian-Pask family with $t_{\lambda^{*}}:=t_{\lambda}^{*}$. Thus there is a canonical $*$-homomorphism $\pi_{q, t}: \mathrm{KP}_{\mathbb{C}}(\Lambda) \rightarrow C^{*}(\Lambda)$ which takes $s_{\lambda} s_{\mu^{*}}$ to $t_{\lambda} t_{\mu}^{*}$.

Proposition 7.3. Suppose that $\Lambda$ is a row-finite $k$-graph without sources. Then $\pi_{q, t}$ is a $*$-isomorphism of $\mathrm{KP}_{\mathbb{C}}(\Lambda)$ onto the $*$-subalgebra

$$
A:=\operatorname{span}\left\{t_{\lambda} t_{\mu}^{*}: \lambda, \mu \in \Lambda\right\} .
$$

To prove this, one reaches for the graded-uniqueness theorem. However, $C^{*}(\Lambda)$ is not graded in the algebraic sense: the subspaces

$$
C^{*}(\Lambda)_{n}:=\overline{\operatorname{span}}\left\{t_{\lambda} t_{\mu}^{*}: d(\lambda)-d(\mu)=n\right\}
$$

satisfy $C^{*}(\Lambda)_{m} C^{*}(\Lambda)_{n} \subset C^{*}(\Lambda)_{m+n}$, and are mutually linearly independent, but they do not span $C^{*}(\Lambda)$ in the usual algebraic sense (see Remark 7.5 below). On the other hand, we have:

Lemma 7.4. The subspaces

$$
A_{n}:=\operatorname{span}\left\{t_{\lambda} t_{\mu}^{*}: d(\lambda)-d(\mu)=n\right\}
$$

form a $\mathbb{Z}^{k}$-grading for the dense subalgebra $A$ of $C^{*}(\Lambda)$.

The proof of the lemma uses the gauge action. For a directed graph $E$, the gauge action is an action of $\mathbb{T}:=\{z \in \mathbb{C}:|z|=1\}$ on $C^{*}(E)$; for a $k$-graph, it is an action $\gamma$ of the $k$-torus $\mathbb{T}^{k}$ on $C^{*}(\Lambda)$. To define $\gamma_{z}$ for $z \in \mathbb{T}^{k}$, invoke the universal property of $\left(C^{*}(\Lambda),(q, t)\right)$ to get a homomorphism $\gamma_{z}: C^{*}(\Lambda) \rightarrow C^{*}(\Lambda)$ such that $\gamma_{z}\left(q_{v}\right)=q_{v}$ and $\gamma_{z}\left(s_{\lambda}\right)=z^{d(\lambda)} s_{\lambda}$, and check that $z \mapsto \gamma_{z}$ is a homomorphism into Aut $C^{*}(\Lambda)$. Then it follows from an $\epsilon / 3$ argument that $\gamma$ is strongly continuous in the sense that $z \mapsto \gamma_{z}(a)$ is continuous for each fixed $a \in C^{*}(\Lambda)$. (The details of the argument are in [28, Proposition 2.1] for $k=1$, and the argument carries over.)

Next we need to integrate continuous functions $f$ on $\mathbb{T}^{k}$ with values in a $C^{*}$ algebra $B$. The easiest way to do this is to represent $B$ faithfully as bounded operators on a Hilbert space $H$, prove that there is a unique bounded operator $T$ on $H$ such that $(T h \mid k)$ is the usual Riemann integral $\int_{\mathbb{T}^{k}}(f(z) h \mid k) d z:=$ $\int_{[0,1]^{k}}\left(f\left(e^{2 \pi i \theta}\right) h \mid k\right) d \theta$ for $h, k \in H$, prove that $T$ belongs to $B$, and then define $\int_{\mathbb{T}^{k}} f(z) d z:=T$. The construction and its properties are described in 28 , 
Lemma 3.1] for the case $k=1$, and the general case is similar. The integral is, for example, linear and norm-decreasing for the sup-norm on $C\left(\mathbb{T}^{k}, B\right)$.

Proof of Lemma 7.4. Since each spanning element $t_{\lambda} t_{\mu}^{*}$ belongs to $A_{d(\lambda)-d(\mu)}$, we can by grouping terms write every $a \in A$ as a finite sum $\sum_{n} a_{n}$ with $a_{n} \in A_{n}$. To see that the $A_{n}$ are independent, suppose that $a_{n} \in A_{n}$ and $\sum_{n} a_{n}=0$. Elementary calculus shows that $\int_{\mathbb{T}^{k}} z^{m} d z$ is 1 if $m=0$ and vanishes otherwise, and hence for $m \in \mathbb{Z}^{k}$ we have

$$
\int_{\mathbb{T}^{k}} z^{-m} \gamma_{z}\left(t_{\lambda} t_{\mu}^{*}\right) d z=\left(\int_{\mathbb{T}^{k}} z^{-m+d(\lambda)-d(\mu)} d z\right) t_{\lambda} t_{\mu}^{*}= \begin{cases}t_{\lambda} t_{\mu}^{*} & \text { if } m=d(\lambda)-d(\mu), \\ 0 & \text { otherwise. }\end{cases}
$$

We deduce from linearity of the integral that if $a_{n} \in A_{n}$, then

$$
\int_{\mathbb{T}^{k}} z^{-m} \gamma_{z}\left(a_{n}\right) d z= \begin{cases}a_{m} & \text { if } m=n \\ 0 & \text { otherwise. }\end{cases}
$$

Now integrating both sides of $\sum_{n} a_{n}=0$ against $z^{-m} \gamma_{z}$ shows that $a_{m}=0$ for all $m$. An application of Lemma 3.3 shows that if $t_{\lambda} t_{\mu}^{*} \in A_{m}$ and $t_{\alpha} t_{\beta}^{*} \in A_{n}$, then $\left(t_{\lambda} t_{\mu}^{*}\right)\left(t_{\alpha} t_{\beta}^{*}\right) \in A_{m+n}$, so $A_{m} A_{n} \subset A_{m+n}$.

Proof of Proposition 7.3. The homomorphism $\pi_{q, t}$ takes $s_{\lambda} s_{\mu^{*}}$ to $t_{\lambda} t_{\mu}^{*}$, hence maps $\mathrm{KP}_{\mathbb{C}}(\Lambda)$ onto $A$ and is graded. Since we know that each $q_{v}$ is nonzero, and since we are working over a field, we have $\pi\left(r p_{v}\right) \neq 0$ for every $r \neq 0$ and every $v \in \Lambda^{0}$. Thus the graded-uniqueness theorem implies that $\pi_{q, t}$ is injective.

Remark 7.5. The gauge action $\gamma$ was crucial in the proof of Lemma 7.4 when we needed to recover the component $a_{m}$ from the expansion $\sum_{n} a_{n}$, so it is certainly connected with the grading. To see why it does not give a grading of the whole $C^{*}$-algebra, consider an action $\beta: \mathbb{T}^{k} \rightarrow$ Aut $B$ of $\mathbb{T}^{k}$ on a $C^{*}$-algebra $B$, and for each $n \in \mathbb{Z}^{k}$, let

$$
B_{n}:=\left\{b \in B: \beta_{z}(b)=z^{n} b \text { for all } z \in \mathbb{T}^{k}\right\} .
$$

Then $B_{n}$ is a closed subspace of $B$, and $E_{n}: b \mapsto b_{n}:=\int_{\mathbb{T}^{k}} z^{-n} \beta_{z}(b) d z$ is a normdecreasing linear operator with range $B_{n}$ satisfying $E_{n} \circ E_{n}=E_{n}$. In the proof of Lemma 7.4. only finitely many $a_{m}$ are nonzero, but in general this is not the case, and we cannot expect to recover every $b \in B$ as a finite sum of elements in the $B_{n}$; the subspaces $B_{n}$ satisfy $B_{m} B_{n} \subset B_{m+n}$, but they do not grade $B$ in the algebraic sense. They are independent (because we can recover $b_{m}$ from a finite sum $\sum_{n} b_{n}$ by integrating), and they do determine $b$ : if $b_{n}=0$ for all $n$, then $b=0$.

One way to see this last point is to represent $B$ faithfully in $B(H)$, and then for each pair $h, k \in H$,

$$
\left(b_{n} h \mid k\right)=\int_{\mathbb{T}^{k}} z^{-n}\left(\beta_{z}(b) h \mid k\right) d z
$$

is the $n$th Fourier coefficient of the continuous function $z \mapsto\left(\beta_{z}(b) h \mid k\right)$. Thus if $b_{n}=0$ for all $n$, all the Fourier coefficients of this function vanish, which implies that $\left(\beta_{z}(b) h \mid k\right)=0$ for all $z, h$ and $k$; taking $z=1$ shows that $(b h \mid k)=0$ for all $h, k$, and $b=0$.

This last argument illustrates the difficulty. If $f$ is smooth, then the Fourier series of $f$ converges uniformly to $f$. When $f$ is just continuous, the Fourier coefficients still determine $f$, but it is not easy to recover $f$ from its Fourier series. 
Remark 7.6. The gauge-invariant uniqueness theorem for $C^{*}(\Lambda)$ says that if $\pi$ : $C^{*}(\Lambda) \rightarrow B$ is a homomorphism (by which we mean a $*$-homomorphism) such that $\pi\left(q_{v}\right) \neq 0$ for all $v$, and if there is a continuous action $\beta$ of $\mathbb{T}^{k}$ on $B$ such that $\pi \circ \gamma_{z}=\beta_{z} \circ \pi$ for every $z \in \mathbb{T}^{k}$, then $\pi$ is injective.

For the gauge action $\gamma$ on $C^{*}(\Lambda)$, we trivially have $A_{n} \subset C^{*}(\Lambda)_{n}$, and since $A$ is dense in $C^{*}(\Lambda)$, the norm continuity of the map $E_{n}: C^{*}(\Lambda) \rightarrow C^{*}(\Lambda)_{n}$ implies that $C^{*}(\Lambda)_{n}$ is as described in (7.1). One can then check that $\pi \circ \gamma_{z}=\beta_{z} \circ \pi$ for every $z \in \mathbb{T}^{k}$ if and only if $\pi\left(C^{*}(\Lambda)_{n}\right) \subset B_{n}$ for every $n \in \mathbb{Z}^{k}$. (In the "if" direction, the continuity of the homomorphisms $\pi \circ \gamma_{z}$ and $\beta_{z} \circ \pi$ allows us to get away with checking equality on the dense subalgebra $A$.) So we could if we wanted reformulate the gauge-invariant uniqueness theorem to look like a graded-uniqueness theorem.

7.2. Rank-2 Bratteli diagrams. Consider a 2-graph $\Lambda$ without sources which is a rank-2 Bratteli diagram in the sense of [25, Definition 4.1]. This means that the blue subgraph $B \Lambda:=\left(\Lambda^{0}, \Lambda^{e_{1}}, r, s\right)$ of the skeleton is a Bratteli diagram in the usual sense, so the vertex set $\Lambda^{0}$ is the disjoint union $\bigsqcup_{n=0}^{\infty} V_{n}$ of finite subsets $V_{n}$, each blue edge goes from some $V_{n+1}$ to $V_{n}$, and the red subgraph $R \Lambda:=\left(\Lambda^{0}, \Lambda^{e_{2}}, r, s\right)$ consists of disjoint cycles whose vertices lie entirely in some $V_{n}$. For each blue edge $e$ there is a unique red edge $f$ with $s(f)=r(e)$, and hence by the factorization property there is a unique blue-red path $\mathcal{F}(e) h$ such that $\mathcal{F}(e) h=f e$. The map $\mathcal{F}: \Lambda^{e_{1}} \rightarrow \Lambda^{e_{1}}$ is a bijection, and induces a permutation of each finite set $\Lambda^{e_{1}} V_{n}$. We write $o(e)$ for the order of $e$ : the smallest $l>0$ such that $\mathcal{F}^{l}(e)=e$.

Proposition 7.7. Suppose that $\Lambda$ is a rank-2 Bratteli diagram. If $\Lambda$ is cofinal and $\left\{o(e): e \in \Lambda^{e_{1}}\right\}$ is unbounded, then $\Lambda$ is aperiodic.

Proposition 7.7 follows from [25, Theorem 5.1], but since [25] uses a different formulation of aperiodicity, we also have to invoke the equivalence of the different notions of aperiodicity [32, Lemma 3.2]. However, the whole point of the finite-path formulation is that it should be easier to verify. So:

Proof of Proposition 7.7. Let $v \in \Lambda^{0}$, say $v \in V_{N_{1}}$, and take $m \neq n$ in $\mathbb{N}^{2}$. If $m_{1} \neq n_{1}$, then any path $\lambda \in v \Lambda^{m \vee n}$ has $\lambda(m) \in V_{N_{1}+m_{1}}$ and $\lambda(n) \in V_{N_{1}+n_{1}}$, and hence satisfies the aperiodicity condition (4.3). So we suppose that $m_{1}=n_{1}$, and without loss of generality that $n_{2}>m_{2}$. As in [25], we further partition each $V_{N}=\bigsqcup_{i=1}^{c_{N}} V_{N, i}$ into the sets of vertices which lie on distinct red cycles.

As in the proof of sufficiency in [25, Theorem 5.1] (see page 158 of [25]), cofinality implies that there exists $N$ such that, for every $M_{1} \geq N, v \Lambda V_{M_{1}, i}$ is nonempty for all $i \leq c_{M_{1}}$, and such that there exist $M \geq \max \left(N, n_{1}+N_{1}\right), i \leq c_{M}$ and $g \in V_{M, i} \Lambda^{e_{1}}$ such that $o(g) \geq n_{2}-m_{2}$. Now choose $\mu \in v \Lambda V_{M, i}$, let $\alpha$ be a red path with vertices in $V_{M, i}, d(\alpha) \geq\left(0, n_{2}\right), r(\alpha)=s(\mu)$ and $s(\alpha)=r(g)$, and take $\lambda:=\mu \alpha g$. Then in particular $d(\lambda) \geq\left(n_{1}, n_{2}\right)=m \vee n$, and $r(\lambda)=v$. We then have

$$
\lambda\left(n+d(\lambda)-(m \vee n)-e_{2}, n+d(\lambda)-(m \vee n)\right)=\lambda\left(d(\lambda)-e_{2}, d(\lambda)\right)=g,
$$

whereas

$$
\begin{aligned}
\lambda\left(m+d(\lambda)-(m \vee n)-e_{2},\right. & m+d(\lambda)-(m \vee n)) \\
= & \lambda\left(d(\lambda)-\left(n_{2}-m_{2}+1\right) e_{2}, d(\lambda)-\left(n_{2}-m_{2}\right)\right) \\
= & \mathcal{F}^{n_{2}-m_{2}}(g),
\end{aligned}
$$

which is not the same as $g$ because $o(g)>n_{2}-m_{2}$. Thus the larger segments in (4.3) cannot be equal, and we have shown that $\Lambda$ is aperiodic. 
Corollary 7.8. Suppose that $\Lambda$ is a rank-2 Bratteli diagram and $K$ is a field. If $\Lambda$ is cofinal and $\left\{o(e): e \in \Lambda^{e_{1}}\right\}$ is unbounded, then $\operatorname{KP}_{K}(\Lambda)$ is simple.

Proof. Since $K$ is a field, basic simplicity is the same as simplicity, so the result follows from Proposition 7.7 and Theorem 5.14

Notice that in the next result we have specialized to the case $K=\mathbb{C}$.

Proposition 7.9. Suppose that $\Lambda$ is a rank-2 Bratteli diagram. If $\Lambda$ is cofinal and $\left\{o(e): e \in \Lambda^{e_{1}}\right\}$ is unbounded, then $\operatorname{KP}_{\mathbb{C}}(\Lambda)$ is not purely infinite in the sense of [4.

Proof. Let $P_{0}:=\sum_{v \in V_{0}} p_{v}$. Since $\mathrm{KP}_{\mathbb{C}}(\Lambda)$ is simple by Corollary 17.8, and since the property of being purely infinite simple passes to corners [2, Proposition 10], it suffices for us to prove that $P_{0} \mathrm{KP}_{\mathbb{C}}(\Lambda) P_{0}$ is not purely infinite. We will show that $P_{0} \mathrm{KP}_{\mathbb{C}}(\Lambda) P_{0}$ does not contain an infinite idempotent. Suppose it does. Then there exist nonzero idempotents $p, p_{1}, p_{2}$ and elements $x, y$ in $P_{0} \operatorname{KP}_{\mathbb{C}}(\Lambda) P_{0}$ such that

$$
p=p_{1}+p_{2}, \quad p_{1} p_{2}=p_{2} p_{1}=0, \quad x y=p \quad \text { and } \quad y x=p_{1} .
$$

Choose $N \in \mathbb{N}$ large enough to ensure that all five elements can be written as linear combinations of elements $s_{\lambda} s_{\mu^{*}}$ for which $s(\lambda)$ and $s(\mu)$ are in $\bigcup_{n=0}^{N} V_{n}$. Then the images of these elements under the isomorphism $\pi_{q, t}$ of Proposition 7.3 all lie in the subalgebra of $P_{0} C^{*}(\Lambda) P_{0}$ spanned by the corresponding $t_{\lambda} t_{\mu}^{*}$, which by [25, Lemma 4.8] is isomorphic to $P_{0} C^{*}\left(\Lambda_{N}\right) P_{0}$, where $\Lambda_{N}$ is the "rank-2 Bratteli diagram of depth $N$ " consisting of all the paths which begin and end in $\bigcup_{n=0}^{N} V_{n}$.

Applying the Kumjian-Pask relations shows that

$$
C^{*}\left(\Lambda_{N}\right)=\overline{\operatorname{span}}\left\{s_{\lambda} s_{\mu}^{*}: s(\lambda)=s(\mu) \in V_{N}\right\} .
$$

If $s(\lambda)=s(\mu)$ and $s(\alpha)=s(\beta)$ lie on different red cycles (that is, belong to different $\left.V_{N, i}\right)$, then $\left(s_{\lambda} s_{\mu}^{*}\right)\left(s_{\alpha} s_{\beta}^{*}\right)=0$, and hence $C^{*}\left(\Lambda_{N}\right)$ is the $C^{*}$-algebraic direct sum of the subalgebras

$$
C_{N, i}=\overline{\operatorname{span}}\left\{s_{\lambda} s_{\mu}^{*}: s(\lambda)=s(\mu) \in V_{N, i}\right\} .
$$

The blue Kumjian-Pask relation implies that the algebras $C_{N, i}$ are unital with identity $P_{i}:=\sum_{\alpha \in \Lambda^{\mathbb{N} e_{1}} V_{N, i}} s_{\alpha} s_{\alpha}^{*}$, and indeed $C_{N, i}=P_{i} C^{*}\left(\Lambda_{N}\right) P_{i}$. Since $P_{i}$ commutes with $P_{0}$, we then have

$$
P_{0} C^{*}\left(\Lambda_{N}\right) P_{0}=\bigoplus_{i=1}^{c_{N}} P_{0} C_{N, i} P_{0} .
$$

The elements $p, p_{1}, p_{2}, x$ and $y$ of $P_{0} C^{*}\left(\Lambda_{N}\right) P_{0}$ all have direct sum decompositions, and the summands all satisfy the relations (7.3); in at least one summand, the component of $p_{2}$ is nonzero, and then the same components of all the rest must be nonzero too. So we may assume that $p, p_{1}, p_{2}, x$ and $y$ all belong to $P_{0} C_{N, i} P_{0}$.

Now consider the subgraph $\Lambda_{N, i}$ of $\Lambda_{N}$ with vertex set $r\left(s^{-1}\left(V_{N, i}\right)\right)$. This 2-graph has sources, but it is locally convex in the sense of [30], and the gauge-invariant uniqueness theorem proved there implies that the inclusion is an isomorphism of $P_{0} C^{*}\left(\Lambda_{N, i}\right) P_{0}$ onto $P_{0} C_{N, i} P_{0}$. The sources in $\Lambda_{N, i}$ all lie on a single red cycle, and hence Lemma 4.5 of [25] implies that $P_{0} C_{N, i} P_{0}$ is isomorphic to $M_{X}(C(\mathbb{T}))=$ $C\left(\mathbb{T}, M_{X}(\mathbb{C})\right)$, where $X$ is the finite set $\Lambda^{N e_{1}} V_{N}=V_{0} \Lambda^{\mathbb{N} e_{1}} V_{N}$. Pulling the five 
elements through all these isomorphisms gives us nonzero idempotents $q, q_{1}, q_{2}$ and elements $f, g$ in $C\left(\mathbb{T}, M_{X}(\mathbb{C})\right)$ such that

$$
q=q_{1}+q_{2}, \quad q_{1} q_{2}=q_{2} q_{1}=0, \quad f g=q \quad \text { and } \quad g f=q_{1} .
$$

Now let $z \in \mathbb{T}$. Then the equations $f(z) g(z)=q(z)$ and $g(z) f(z)=q_{1}(z)$ imply that $g(z)$ is an isomorphism of $q(z) \mathbb{C}^{X}$ onto $q_{1}(z) \mathbb{C}^{X}$, so the matrices $q(z)$ and $q_{1}(z)$ have the same rank. On the other hand, since $q_{1}(z)$ and $q_{2}(z)$ are orthogonal, $\operatorname{rank}\left(q_{1}(z)+q_{2}(z)\right)=\operatorname{rank} q_{1}(z)+\operatorname{rank} q_{2}(z)$. Now $q=q_{1}+q_{2}$ implies that $\operatorname{rank} q_{2}(z)=0$ for all $z$, which contradicts the assumption that $p_{2}$ is nonzero. Thus there is no infinite idempotent in $P_{0} \mathrm{KP}_{\mathbb{C}}(\Lambda) P_{0}$, as claimed. Thus $P_{0} \mathrm{KP}_{\mathbb{C}}(\Lambda) P_{0}$ is not purely infinite, and neither is $\operatorname{KP}_{\mathbb{C}}(\Lambda)$.

Rank-2 Bratteli diagrams were invented in 25 to prove that the dichotomy of 22. for simple graph $C^{*}$-algebras does not extend to the $C^{*}$-algebras of higher-rank graphs. We can now use them to see that the dichotomy of [3, Theorem 4.4] for simple Leavitt path algebras does not extend either.

Theorem 7.10. Suppose that $\Lambda$ is a rank-2 Bratteli diagram, that $\Lambda$ is cofinal, and that $\left\{o(e): e \in \Lambda^{e_{1}}\right\}$ is unbounded. Then $\mathrm{KP}_{\mathbb{C}}(\Lambda)$ is simple but is neither purely infinite nor locally matricial.

Proof. Corollary 7.8 implies that $\mathrm{KP}_{\mathbb{C}}(\Lambda)$ is simple, and Proposition 7.9 that it is not purely infinite. To see that it is not locally matricial, consider the element $s_{\mu}$ associated to a single red cycle $\mu$. Since $v:=r(\mu)=s(\mu)$ receives just one red path of length $|\mu|$, namely $\mu$, the Kumjian-Pask relation (KP4) at $v$ for $n=|\mu| e_{2}$ (which only involves red paths) says that $p_{v}=s_{\mu} s_{\mu}^{*}$. Thus if $E$ is the directed graph consisting of a single vertex $w$ and a single loop $e$ at $w$ and $(p, s)$ is the universal Kumjian-Pask $\Lambda$-family in $\operatorname{KP}_{\mathbb{C}}(\Lambda)$, then there is a homomorphism $\pi$ of the Leavitt path algebra $L_{\mathbb{C}}(E)$ into $\mathrm{KP}_{\mathbb{C}}(\Lambda)$ which takes $w$ to $p_{w}$, e to $s_{\mu}$ and $e^{*}$ to $s_{\mu^{*}}$. Since the image algebra $A$ is graded by $A_{m}:=A \cap \operatorname{KP}_{\mathbb{C}}(\Lambda)_{m|\mu|}=\operatorname{span}\left\{\mu^{m}\right\}$, and since $p_{w} \neq 0$, the graded-uniqueness theorem for ordinary graphs implies that $\pi$ is injective. But $e$ generates the infinite-dimensional algebra $L_{\mathbb{C}}(E)=\mathbb{C}\left[x, x^{-1}\right]$, so $s_{\mu}$ does not lie in a finite-dimensional subalgebra.

Remark 7.11. The main examples of rank-2 Bratteli diagrams are the families $\left\{\Lambda_{\theta}: \theta \in(0,1) \backslash \mathbb{Q}\right\}$ in [25, Example 6.5] and $\{\Lambda(\mathbf{m}): \mathbf{m}$ is supernatural $\}$ in [25, Example 6.7]. These provide models for two important families of $C^{*}$-algebras called the irrational rotation algebras $A_{\theta}$ and the Bunce-Deddens algebras $\operatorname{BD}(\mathbf{m})$. That their $C^{*}$-algebras satisfy $C^{*}\left(\Lambda_{\theta}\right) \cong A_{\theta}$ and $C^{*}(\Lambda(\mathbf{m})) \cong \mathrm{BD}(\mathbf{m})$ is proved in 25] by showing that the graph algebras are AT-algebras with real rank zero, hence fall into the class of $C^{*}$-algebras covered by a classification theorem of Elliott [15, computing their $K$-theory, and comparing this $K$-theory with the known $K$-theory of $A_{\theta}$ and $\mathrm{BD}(\mathbf{m})$. So the proofs will not carry over to Kumjian-Pask algebras.

\section{REFERENCES}

[1] G. Abrams, G. Aranda Pino, The Leavitt path algebra of a graph, J. Algebra 293 (2005), 319-334. MR2172342(2007b:46085)

[2] G. Abrams, G. Aranda Pino, Purely infinite simple Leavitt path algebras, J. Pure Appl. Algebra 207 (2007), 553-563. MR2265539 (2007f:16062)

[3] G. Abrams, G. Aranda Pino, The Leavitt path algebras of arbitrary graphs, Houston J. Math. 34 (2008), 423-442. MR2417402(2009h:16043) 
[4] P. Ara, K.R. Goodearl, E. Pardo, $K_{0}$ of purely infinite simple regular rings, K-Theory 26 (2002), 69-100. MR1918211 (2004e:19001)

[5] P. Ara, M.A. Moreno, E. Pardo, Nonstable K-theory for graph algebras, Algebr. Represent. Theory 10 (2007), 157-178. MR2310414 (2008b:46094)

[6] G. Aranda Pino, K. Crow, The center of a Leavitt path algebra, Rev. Mat. Iberoam. 27 (2011), 621-644. MR2848533 (2012h:16057)

[7] G. Aranda Pino, K.R. Goodearl, F. Perera, M. Siles Molina, Non-simple purely infinite rings, Amer. J. Math. 132 (2010), 563-610. MR2666902(2011e:16064)

[8] T. Bates, J.H. Hong, I. Raeburn, W. Szymański, The ideal structure of the $C^{*}$-algebras of infinite graphs, Illinois J. Math. 46 (2002), 1159-1176. MR.1988256 (2004i:46105)

[9] T. Bates, D. Pask, I. Raeburn, W. Szymański, The $C^{*}$-algebras of row-finite graphs, New York J. Math. 6 (2000), 307-324. MR.1777234 (2001k:46084)

[10] B. Blackadar, Shape theory for $C^{*}$-algebras, Math. Scand. 56 (1985), 249-275. MR 813640 (87b:46074)

[11] J. Cuntz, A class of $C^{*}$-algebras and topological Markov chains II, Invent. Math. 63 (1981), 25-40. MR608527 (82f:46073b)

[12] J. Cuntz, W. Krieger, A class of $C^{*}$-algebras and topological Markov chains, Invent. Math. 56 (1980), 251-268. MR.561974 (82f:46073a)

[13] K.R. Davidson, S.C. Power, D. Yang, Dilation theory for rank 2 graph algebras, J. Operator Theory 63 (2010), 245-270. MR2651911(2011i:47110)

[14] K.R. Davidson, D. Yang, Periodicity in rank 2 graph algebras, Canad. J. Math. 61 (2009), 1239-1261. MR2588421 (2010h:46077)

[15] G.A. Elliott, On the classification of $C^{*}$-algebras of real rank zero, J. Reine Angew. Math. 443 (1993), 179-219. MR.1241132(94i:46074)

[16] N.J. Fowler, A. Sims, Product systems over right-angled Artin semigroups, Trans. Amer. Math. Soc. 354 (2002), 1487-1509. MR1873016 (2002j:18006)

[17] R. Hazlewood, I. Raeburn, A. Sims, S.B.G. Webster, On some fundamental results about higher-rank graphs and their $C^{*}$-algebras, to appear in Proc. Edinb. Math. Soc.

[18] J.H. Hong, W. Szymański, The primitive ideal space of the $C^{*}$-algebras of infinite graphs, $J$. Math. Soc. Japan 56 (2004), 45-64. MR2023453 (2004j:46088)

[19] A. an Huef, I. Raeburn, The ideal structure of Cuntz-Krieger algebras, Ergodic Theory Dynam. Systems 17 (1997), 611-624. MR.1452183 (98k:46098)

[20] D.W. Kribs, S.C. Power, The analytic algebras of higher rank graphs, Math. Proc. Royal Irish Acad. 106A (2006), 199-218. MR2266827(2007h:47129)

[21] A. Kumjian, D. Pask, Higher rank graph $C^{*}$-algebras, New York J. Math. 6 (2000), 1-20. MR.1745529 (2001b:46102)

[22] A. Kumjian, D. Pask, I. Raeburn, Cuntz-Krieger algebras of directed graphs, Pacific J. Math. 184 (1998), 161-174. MR 1626528 (99i:46049)

[23] A. Kumjian, D. Pask, I. Raeburn, J. Renault, Graphs, groupoids, and Cuntz-Krieger algebras, J. Funct. Anal. 144 (1997), 505-541. MR1432596 (98g:46083)

[24] P. Lewin, A. Sims, Aperiodicity and cofinality for finitely aligned higher-rank graphs, Math. Proc. Camb. Phil. Soc. 149 (2010), 333-350. MR2670219

[25] D. Pask, I. Raeburn, M. Rørdam, A. Sims, Rank-two graphs whose $C^{*}$-algebras are direct limits of circle algebras, J. Funct. Anal. 239 (2006), 137-178. MR2258220(2007e:46053)

[26] D. Pask, I. Raeburn, N.A. Weaver, A family of 2-graphs arising from two-dimensional subshifts, Ergodic Theory Dynam. Systems 29 (2009), 1613-1639. MR2545020 (2010g:46115)

[27] S.C. Power, Classifying higher rank analytic Toeplitz algebras, New York J. Math. 13 (2007), 271-298. MR2336241 (2010b:47228)

[28] I. Raeburn, Graph Algebras, CBMS Regional Conference Series in Mathematics, vol. 103, Amer. Math. Soc., Providence, RI, 2005. MR2135030 (2005k:46141)

[29] I. Raeburn, Chapter 1 in: Graph Algebras: Bridging the Gap between Analysis and Algebra, edited by G. Aranda Pino, F. Perera Domènech and M. Siles Molina, University of Malaga, 2006, pages 1-21. MR2135030 (2005k:46141)

[30] I. Raeburn, A. Sims, T. Yeend, Higher-rank graphs and their $C^{*}$-algebras, Proc. Edinburgh Math. Soc. 46 (2003), 99-115. MR.1961175 (2004f:46068)

[31] I. Raeburn, A. Sims, T. Yeend, The $C^{*}$-algebras of finitely aligned higher-rank graphs, $J$. Funct. Anal. 213 (2004), 206-240. MR2069786 (2005e:46103) 
[32] D.I. Robertson, A. Sims, Simplicity of $C^{*}$-algebras associated to higher-rank graphs, Bull. London Math. Soc. 39 (2007), 337-344. MR2323468 (2008g:46099)

[33] G. Robertson, T. Steger, $C^{*}$-algebras arising from group actions on the boundary of a triangle building, Proc. London Math. Soc. 72 (1996), 613-637. MR1376771 (98b:46088)

[34] G. Robertson, T. Steger, Affine buildings, tiling systems and higher-rank Cuntz-Krieger algebras, J. Reine Angew. Math. 513 (1999), 115-144. MR1713322 (2000j:46109)

[35] A. Sims, Lecture notes on higher-rank graphs and their $C^{*}$-algebras, Notes for Summer School on $C^{*}$-algebras and their interplay with dynamics, Nordfjordheid, Norway, 2010.

[36] A. Skalski, J. Zacharias, Entropy of shifts on higher-rank graph $C^{*}$-algebras, Houston J. Math. 34 (2008), 269-282. MR2383707(2009j:46165)

[37] M. Tomforde, Uniqueness theorems and ideal structure for Leavitt path algebras, J. Algebra 318 (2007), 270-299. MR2363133 (2008h:16030)

[38] M. Tomforde, Leavitt path algebras with coefficients in a commutative ring, J. Pure Appl. Algebra 215 (2011), 471-484. MR2738365

[39] D. Yang, Endomorphisms and modular theory of 2-graph $C^{*}$-algebras, Indiana Univ. Math. J. 59 (2010), 495-520. MR2648076

Departamento de Álgebra, Geometría y Topología, Universidad de Málaga, 29071 MÁlaga, SPAIN

E-mail address: g.aranda@uma.es

Department of Mathematics and Statistics, University of Otago, PO Box 56, Dunedin 9054, New Zealand

E-mail address: jclark@maths.otago.ac.nz

Department of Mathematics and Statistics, University of Otago, PO Box 56, Dunedin 9054, NEW ZEALAND

E-mail address: astrid@maths.otago.ac.nz

Department of Mathematics and Statistics, University of Otago, PO Box 56, Dunedin 9054, New ZEALAND

E-mail address: iraeburn@maths.otago.ac.nz 